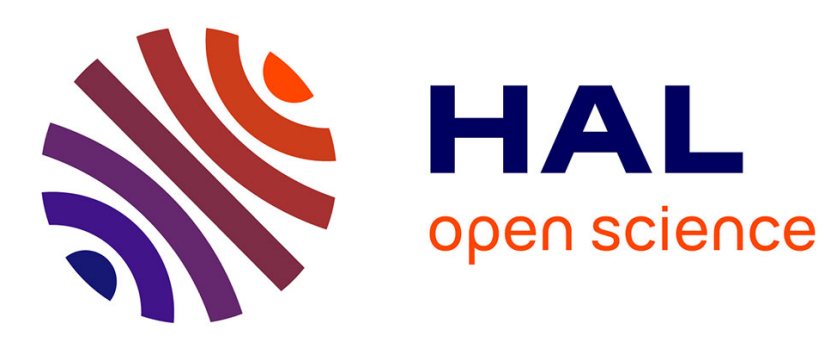

\title{
Enhanced widely linear filtering to make quasi-rectilinear signals almost equivalent to rectilinear ones for SAIC/MAIC
}

Pascal Chevalier, Rémi Chauvat, Jean-Pierre Delmas

\section{- To cite this version:}

Pascal Chevalier, Rémi Chauvat, Jean-Pierre Delmas. Enhanced widely linear filtering to make quasirectilinear signals almost equivalent to rectilinear ones for SAIC/MAIC. IEEE Transactions on Signal Processing, 2018, 66 (6), pp.1438 - 1453. 10.1109/TSP.2017.2784403 . hal-01702741

\section{HAL Id: hal-01702741 \\ https://hal.science/hal-01702741}

Submitted on 7 Feb 2018

HAL is a multi-disciplinary open access archive for the deposit and dissemination of scientific research documents, whether they are published or not. The documents may come from teaching and research institutions in France or abroad, or from public or private research centers.
L'archive ouverte pluridisciplinaire HAL, est destinée au dépôt et à la diffusion de documents scientifiques de niveau recherche, publiés ou non, émanant des établissements d'enseignement et de recherche français ou étrangers, des laboratoires publics ou privés. 


\title{
Enhanced Widely Linear Filtering to Make Quasi-Rectilinear Signals Almost Equivalent to Rectilinear Ones for SAIC/MAIC
}

\author{
Pascal Chevalier, Rémi Chauvat and Jean-Pierre Delmas, Senior Member, IEEE
}

\begin{abstract}
Widely linear (WL) receivers have the capability to perform single antenna interference cancellation (SAIC) of one rectilinear $(\mathbf{R})$ or quasi-rectilinear $(Q R)$ co-channel interference (CCI), a function which is operational in global system for mobile communications (GSM) handsets in particular. Moreover, SAIC technology for QR signals is still required for voice services over adaptive multi-user channels on one slot (VAMOS) standard, a recent evolution of GSM/EDGE standard, to mitigate legacy GSM CCI in particular. It is also required for filter bank multi-carrier offset quadrature amplitude modulation (FBMCOQAM) networks, which are candidate for $5 \mathrm{G}$ mobile networks, to mitigate inter-carrier interference (ICI) at reception for frequency selective propagation channels in particular. In this context, the purpose of this paper is twofold. The first one is to get more insights into the existing SAIC technology, and its extension to multiple antenna called MAIC, by showing analytically that, contrary to what is accepted as true in the literature, SAIC/MAIC implemented from standard WL filtering may be less efficient for $Q R$ signals than for $R$ ones. From this result, the second purpose of the paper is to propose and to analyze, for QR signals and frequency selective fading channels, a SAIC/MAIC enhancement based on a three-input WL frequency shift (FRESH) receiver, making QR signals always almost equivalent to $R$ ones for WL filtering in the presence of CCI. The results of the paper, completely new, may contribute to develop elsewhere new powerful WL receivers for $Q R$ signals and for both VAMOS and FBMC-OQAM networks in particular.
\end{abstract}

Index Terms-Non-Circular, Widely Linear, Single Antenna Interference Cancellation (SAIC), Rectilinear, Quasi-Rectilinear, CCI, Continous-Time, Pseudo-MLSE, FRESH, MSK, GMSK, OQAM, ASK, VAMOS, FBMC

\section{INTRODUCTION}

$\mathbf{S}$ INCE two decades and the pioneer works on the subject [1-4], WL filtering has raised up a great interest for second-order (SO) non-circular (or improper) signals [5], in numerous areas. Nevertheless, the application which has received the greatest interest is CCI mitigation in radiocommunication networks using $\mathrm{R}$ or $\mathrm{QR}$ modulations. Let us recall that $\mathrm{R}$ modulations correspond to mono-dimensional modulations such as amplitude modulation (AM), amplitude shift keying (ASK) or binary phase shift keying (BPSK) modulations, whereas QR modulations are complex modulations corresponding, after a simple derotation operation [6], to a complex filtering of a $\mathrm{R}$ modulation. Examples of $\mathrm{QR}$ modulations are $\pi / 2$-BPSK, minimum shift keying (MSK) or OQAM modulations, while an example of approximated QR modulation is the Gaussian MSK (GMSK) modulation. One of the most important properties of WL filtering is its capability to perform SAIC of one R or QR multi-user CCI, allowing the separation of two users from only one receive antenna [79]. The effectiveness of this technology jointly with its low complexity explain why it is currently operational in most of GSM handsets, allowing significant network's capacity gains for the GSM system [9], [10]. Extension of the SAIC concept to a multi-antenna reception is called MAIC and has been of great interest for GPRS networks in particular [11]. Other works about the SAIC/MAIC concepts by WL filtering are presented in [12-15].

Despite the important development of $3 \mathrm{G}$ and $4 \mathrm{G}$ mobile cellular networks all over the world for data and video traffic, there is still a significant development of GSM/EDGE networks and their evolutions in emerging markets such as China, India, Africa and Eastern Europe [16]. To accommodate the growing voice traffic and also to make room for increasing data traffic, there is a necessity to increase the spectral efficiency of speech services. For this reason, a new technology, called VAMOS, has been recently standardized [16]. The aim of VAMOS is to increase the capacity of GSM, while maintaining backward compatibility with the legacy system. VAMOS enables the transmission of two GSM voice streams on the same TDMA slot at the same carrier frequency through the so-called orthogonal sub channel (OSC) multiple access technique which aims at doubling the number of users served by a cell. The separation, at the handset level, of the two streams, distorted by frequency selective channels and potentially corrupted by co-channel OSC and/or legacy GMSK interference, coming from out of cell OSC and/or legacy base-stations, requires the implementation of enhanced SAIC techniques for QR signals [16]. Such preliminary enhanced techniques for VAMOS, based on standard WL filtering, have been proposed recently in [17-19] for SAIC and in [20] for SAIC/MAIC, for both OSC downlink and uplink transmissions respectively.

Moreover, 4G networks using LTE [21] or LTE-Advanced [22] technologies employ multiple input multiple output (MIMO) orthogonal frequency division multiplex (OFDM) for transmission in the downlink. In order to avoid frequency planning, a frequency reuse factor of one may be possible, which requires receivers robust to CCI. For this reason, two adaptations of the SAIC/MAIC concept to OFDM transmissions using $\mathrm{R}$ modulations have been presented in [23] and [24] for SISO/SIMO and MISO/MIMO systems using the Alamouti scheme respectively. However OFDM waveforms are not well-localized in frequency and require strong timefrequency synchronization constraints, which is not compatible with the needs of the $5 \mathrm{G}$ wireless networks such as a high density of device-to-device or machine-to-machine links [25]. For these reasons, filtered multi-carrier waveforms such as FBMC waveforms [26], which are well localized in frequency 
and compatible with asynchronous links, are considered as good candidates for $5 \mathrm{G}$ networks. The coupling of FBMC waveforms with OQAM modulation, giving rise to FBMCOQAM waveforms [27], has been shown to maximize the spectral efficiency while removing the ICI induced by the filtering operation for SISO/SIMO links in flat fading channels [27]. However, for frequency-selective channels or for MIMO links, FBMC-OQAM waveforms still generate ICI at reception. As the ICI associated with a given subcarrier is a frequency shifted QR interference, it may be removed effectively by WL filtering. Besides, as an FBMC-OQAM $\mathrm{CCI}$ is the sum of frequency shifted QR signals, it is a SO non-circular CCI as shown in [28]. Enhanced SAIC/MAIC techniques aiming at removing both inter-symbol interference (ISI), ICI and potential CCI are thus also required for FBMCOQAM networks in particular. Preliminary standard WL based solutions are presented in [29-31] for MIMO links using spatial multiplexing at transmission and in [32], [33] for SISO links. Reference [32] concerns CCI mitigation in flat fading channels, while [33] deals with both ISI and ICI mitigation in frequency selective channel.

Thus, as a summary, at least for VAMOS/OSC and FBMCOQAM networks, which both use QR modulations, enhanced SAIC/MAIC techniques are still required. In this context, the purpose of this paper is twofold. The first one is to get more insights into the existing SAIC/MAIC technology by proving analytically, which is completely original, that, contrary to what is implicitly accepted as true in the literature [6], [7], [9], [17-20], [29-31], [34-36], QR signals may be less efficient than R ones for SAIC/MAIC implemented from some standard WL filtering. Starting from this result, the second purpose of the paper is to propose and to analyse, partially analytically, which is also very original, for QR signals and frequency selective fading channels, an enhanced SAIC/MAIC technique based on a three-input WL FRESH receiver. This new technique makes QR signals always almost equivalent to $\mathrm{R}$ ones for WL filtering in the presence of CCI.

To compare $\mathrm{QR}$ and $\mathrm{R}$ signals for SAIC/MAIC from standard WL filtering and to show the effectiveness of the proposed enhanced SAIC/MAIC technique for QR signals, we adopt a continuous-time (CT) approach. The choice of such an approach here is justified by three reasons. The first one is that the implementation issues are out of the scope of the paper, which is mainly conceptual. The second one is that a CT approach allows us to remove both the filtering structure constraints imposed by a discrete-time (DT) approach and the potential influence of the sample rate. The third one, is that it allows us to obtain analytical and interpretable expressions for the performance at the output of all the linear and WL receivers considered in this paper, which is completely original. Besides, we choose a pseudo maximum likelihood sequence estimation (pseudo-MLSE) approach, much more easy to derive than an MLSE approach and much more powerful than a minimum mean square error (MMSE) approach. Note that the results of the paper may contribute to develop elsewhere alternative powerful WL receivers for QR signals and for both VAMOS and FBMC-OQAM networks in particular. Note that preliminary results of the paper have been introduced briefly in the conference papers [37] and [38].

Let us recall that WL FRESH filtering has already been used these two last decades for applications such as MMSE estimation [2], beamforming [39] or properization of improper cyclostationary signals [40]. Moreover, WL FRESH filtering for equalization/demodulation purposes in the presence of CCI has been considered in [41-44] for R signals and in [4547] for QR signals. However, while [45] concerns DS-CDMA systems, [47] considers a particular DT MMSE approach and assumes different cyclostationarity properties of the signal of interest (SOI) and CCI. Besides, [46] mentions the proposed enhanced SAIC/MAIC technique for CCI cancellation in the GSM context but through a DT approach at the symbol rate, which finally reduces to the standard SAIC/MAIC approach. Finally, to the best of our knowledge, analytical performance at the output of a FRESH receiver in the presence of a CCI have never been computed before.

The paper is organized as follows. Section II introduces the observation model and the extended one for standard WL processing of both $\mathrm{R}$ and $\mathrm{QR}$ signals, jointly with the SO statistics of the total noise. Section III introduces the conventional linear and standard WL pseudo-MLSE receivers for the demodulation of $\mathrm{R}$ and $\mathrm{QR}$ signals in the presence of multi-user CCI. Section IV presents, for several propagation channels, in the presence of one CCI and in terms of output signal to interference plus noise ratio (SINR) on the current symbol, a comparative performance analysis of SAIC/MAIC from standard WL pseudo-MLSE receivers for both $\mathrm{R}$ and $\mathrm{QR}$ signals. Section $\mathrm{V}$ introduces, for $\mathrm{QR}$ signals, the enhanced SAIC/MAIC concept from the three-input WL FRESH receiver and analyzes its performance, in terms of output SINR on the current symbol, in the presence of one CCI. Section VI analyzes some complexity issues of the two and three-input pseudo-MLSE receivers for QR signals and shows that the results obtained through the output SINR criterion are still valid for the output symbol error rate (SER). Finally section VII concludes this paper.

Notations: Before proceeding, we fix the notations used throughout the paper. Non boldface symbols are scalar whereas lower (upper) case boldface symbols denote column vectors (matrices). $(.)^{T},(.)^{H}$ and $(.)^{*}$ means the transpose, conjugate transpose and conjugate, respectively. $\mathbf{0}_{K}$ and $\mathbf{I}_{K}$ are the zero and the identity matrices of dimension $K$ respectively. $\delta(x)$ is the Kronecker symbol such that $\delta(x)=1$ for $x=0$ and $\delta(x)=0$ for $x \neq 0$. Moreover, all Fourier transforms of vectors $\mathbf{x}$ and matrices $\mathbf{X}$ use the same notation where $t$ or $\tau$ is simply replaced by $f$.

\section{Models and Total Noise Second-Order STATISTICS}

\section{A. Observation model and total noise SO statistics}

We consider an array of $N$ narrow-band antennas receiving the contribution of a SOI, which may be R or QR, and a total noise. The $N \times 1$ vector of complex amplitudes of the data at the output of these antennas after frequency synchronization can then be written as

$$
\mathbf{x}(t)=\sum_{k} a_{k} \mathbf{g}(t-k T)+\mathbf{n}(t)
$$


Here, $a_{k}=b_{k}$ for $\mathrm{R}$ signals whereas $a_{k}=j^{k} b_{k}$ for QR signals, where $b_{k}$ are real-valued zero-mean independent identically distributed (i.i.d.) random variables, corresponding to the SOI symbols for R signals and directly related to the SOI symbols for QR signals [34], [48], [49], $T$ is the symbol period for R, $\pi / 2$-BPSK, MSK and GMSK signals [48], [49] and half the symbol period for OQAM signals [34], $\mathbf{g}(t)=v(t) \otimes \mathbf{h}(t)$ is the $N \times 1$ impulse response of the SOI global channel, $\otimes$ is the convolution operation, $v(t)$ and $\mathbf{h}(t)$ are respectively the scalar and $N \times 1$ impulse responses of the SOI pulse shaping filter and propagation channel respectively and $\mathbf{n}(t)$ is the $N \times 1$ zero-mean total noise vector. Note that model (1) with $a_{k}=j^{k} b_{k}$ is exact for $\pi / 2$-BPSK, MSK and OQAM signals whereas it is only an approximated model for GMSK signals [48].

The SO statistics of $\mathbf{n}(t)$ are characterized by the two correlation matrices $\mathbf{R}_{n}(t, \tau)$ and $\mathbf{C}_{n}(t, \tau)$, defined by

$$
\begin{aligned}
& \mathbf{R}_{n}(t, \tau) \triangleq \mathrm{E}\left[\mathbf{n}\left(t+\frac{\tau}{2}\right) \mathbf{n}^{H}\left(t-\frac{\tau}{2}\right)\right], \\
& \mathbf{C}_{n}(t, \tau) \triangleq \mathrm{E}\left[\mathbf{n}\left(t+\frac{\tau}{2}\right) \mathbf{n}^{T}\left(t-\frac{\tau}{2}\right)\right],
\end{aligned}
$$

We assume that $\mathbf{n}(t)$ is composed of circular, stationary, temporally and spatially white background noise and multiuser CCI coming from the same network, and then having the same nature ( $\mathrm{R}$ or $\mathrm{QR})$, the same symbol period and the same pulse-shaping filter as the SOI. Note that the analysis of the impact of CCI having a symbol period or a pulse shaping filter different from that of the SOI is out of the scope of the paper. Under the previous assumptions, it is easy to verify that $\mathbf{R}_{n}(t, \tau)$ and $\mathbf{C}_{n}(t, \tau)$ are periodic functions of $t$, whose periods are equal to $T$ and $T$ respectively for R signals, and to $T$ and $2 T$ respectively for $\mathrm{QR}$ signals. Matrices $\mathbf{R}_{n}(t, \tau)$ and $\mathbf{C}_{n}(t, \tau)$ have then Fourier series expansions given by

$$
\begin{aligned}
& \mathbf{R}_{n}(t, \tau)=\sum_{\alpha_{i}} \mathbf{R}_{n}^{\alpha_{i}}(\tau) e^{j 2 \pi \alpha_{i} t}, \\
& \mathbf{C}_{n}(t, \tau)=\sum_{\beta_{i}} \mathbf{C}_{n}^{\beta_{i}}(\tau) e^{j 2 \pi \beta_{i} t} .
\end{aligned}
$$

Here $\alpha_{i}$ and $\beta_{i}$ are the so-called non-conjugate and conjugate SO cyclic frequencies of $\mathbf{n}(t)$ such that $\alpha_{i}=\beta_{i}=i / T(i \in \mathbb{Z})$ for $\mathrm{R}$ signals and $\alpha_{i}=i / T$ and $\beta_{i}=(2 i+1) / 2 T(i \in$ $\mathbb{Z}$ ) for QR signals [50-52], $\mathbf{R}_{n}^{\alpha_{i}}(\tau)$ and $\mathbf{C}_{n}^{\beta_{i}}(\tau)$ are the first and second cyclic correlation matrices of $\mathbf{n}(t)$ for the cyclic frequencies $\alpha_{i}$ and $\beta_{i}$ and the delay $\tau$, defined by

$$
\begin{aligned}
\mathbf{R}_{n}^{\alpha_{i}}(\tau) & \triangleq\left\langle\mathbf{R}_{n}(t, \tau) e^{-j 2 \pi \alpha_{i} t}\right\rangle_{\infty}, \\
\mathbf{C}_{n}^{\beta_{i}}(\tau) & \triangleq\left\langle\mathbf{C}_{n}(t, \tau) e^{-j 2 \pi \beta_{i} t}\right\rangle_{\infty},
\end{aligned}
$$

where $\langle\cdot\rangle_{\infty}$ is the temporal mean operation in $t$ over an infinite observation duration. The Fourier transforms, $\mathbf{R}_{n}^{\alpha_{i}}(f)$ and $\mathbf{C}_{n}^{\beta_{i}}(f)$, of $\mathbf{R}_{n}^{\alpha_{i}}(\tau)$ and $\mathbf{C}_{n}^{\beta_{i}}(\tau)$ respectively, are called the first and second cyclospectrum of $\mathbf{n}(t)$ for the cyclic frequencies $\alpha_{i}$ and $\beta_{i}$, respectively. Note that the first and second cyclospectrum of the transmitted SOI,

$$
s(t) \triangleq \sum_{k} a_{k} v(t-k T),
$$

for the cyclic frequencies $\alpha_{i}$ and $\beta_{i}$, respectively, denoted by $\mathrm{r}_{s}^{\alpha_{i}}(f)$ and $\mathrm{c}_{s}^{\beta_{i}}(f)$ respectively, are given, after elementary computations, for both R and QR SOI, by the expressions

$$
\begin{aligned}
\mathrm{r}_{s}^{\alpha_{i}}(f) & =\frac{\pi_{b}}{T} v\left(f+\frac{\alpha_{i}}{2}\right) v^{*}\left(f-\frac{\alpha_{i}}{2}\right), \\
\mathrm{c}_{s}^{\beta_{i}}(f) & =\frac{\pi_{b}}{T} v\left(f+\frac{\beta_{i}}{2}\right) v\left(\frac{\beta_{i}}{2}-f\right),
\end{aligned}
$$

where $\pi_{b} \triangleq \mathrm{E}\left[b_{k}^{2}\right]$.

\section{B. Extended two-input models for standard WL processing}

For both $\mathrm{R}$ and $\mathrm{QR}$ signals, a conventional linear processing of $\mathbf{x}(t)$ only exploits the information contained at the zero non-conjugate $(\alpha=0)$ SO cyclic frequency of $\mathbf{x}(t)$, through the exploitation of the temporal mean of the first correlation matrix, $\mathbf{R}_{x}(t, \tau) \triangleq \mathrm{E}\left[\mathbf{x}(t+\tau / 2) \mathbf{x}^{H}(t-\tau / 2)\right]$, of $\mathbf{x}(t)$.

For $\mathrm{R}$ signals, a standard WL processing of $\mathbf{x}(t)$, i.e. a linear processing of $\widetilde{\mathbf{x}}(t) \triangleq\left[\mathbf{x}^{T}(t), \mathbf{x}^{H}(t)\right]^{T}$, only exploits the information contained at the zero non-conjugate and conjugate $(\alpha, \beta)=(0,0)$ SO cyclic frequencies of $\mathbf{x}(t)$ through the exploitation of the temporal mean of the first correlation matrix, $\mathbf{R}_{\widetilde{x}}(t, \tau) \triangleq \mathrm{E}\left[\widetilde{\mathbf{x}}(t+\tau / 2) \widetilde{\mathbf{x}}^{H}(t-\tau / 2)\right]$, of the extended, or two-input model

$$
\widetilde{\mathbf{x}}(t) \triangleq\left[\mathbf{x}^{T}(t), \mathbf{x}^{H}(t)\right]^{T}=\sum_{k} b_{k} \widetilde{\mathbf{g}}(t-k T)+\widetilde{\mathbf{n}}(t),
$$

where $\widetilde{\mathbf{g}}(t) \triangleq\left[\mathbf{g}^{T}(t), \mathbf{g}^{H}(t)\right]^{T}$ and $\widetilde{\mathbf{n}}(t) \triangleq\left[\mathbf{n}^{T}(t), \mathbf{n}^{H}(t)\right]^{T}$.

For QR signals, as no information is contained at $\beta=0$, a derotation preprocessing of the data is required before standard WL filtering. Using (1) for QR signals, the derotated observation vector can be written as

$$
\mathbf{x}_{d}(t) \triangleq j^{-\frac{t}{T}} \mathbf{x}(t)=\sum_{k} b_{k} \mathbf{g}_{d}(t-k T)+\mathbf{n}_{d}(t),
$$

where $\mathbf{g}_{d}(t) \triangleq j^{-t / T} \mathbf{g}(t)$ and $\mathbf{n}_{d}(t) \triangleq j^{-t / T} \mathbf{n}(t)$. Expression (12) shows that the derotation operation makes a QR signal looks like a $\mathrm{R}$ signal, with a non-zero information at the zero conjugate SO cyclic frequency. Indeed, it is easy to verify that the two correlation matrices, $\mathbf{R}_{x_{d}}(t, \tau) \triangleq \mathrm{E}\left[\mathbf{x}_{d}(t+\right.$ $\left.\tau / 2) \mathbf{x}_{d}^{H}(t-\tau / 2)\right]$ and $\mathbf{C}_{x_{d}}(t, \tau) \triangleq \mathrm{E}\left[\mathbf{x}_{d}(t+\tau / 2) \mathbf{x}_{d}^{T}(t-\tau / 2)\right]$ of $\mathbf{x}_{d}(t)$ are such that

$$
\begin{aligned}
& \mathbf{R}_{x_{d}}(t, \tau)=j^{-\frac{\tau}{T}} \mathbf{R}_{x}(t, \tau), \\
& \mathbf{C}_{x_{d}}(t, \tau)=j^{-\frac{2 t}{T}} \mathbf{C}_{x}(t, \tau) \triangleq e^{-j \frac{2 \pi t}{2 T}} \mathbf{C}_{x}(t, \tau),
\end{aligned}
$$

where $\mathbf{C}_{x}(t, \tau) \triangleq \mathrm{E}\left[\mathbf{x}(t+\tau / 2) \mathbf{x}^{T}(t-\tau / 2)\right]$. These expressions show that the non-conjugate, $\alpha_{d_{i}}$, and conjugate, $\beta_{d_{i}}$, SO cyclic frequencies of $\mathbf{x}_{d}(t)$ are such that $\alpha_{d_{i}}=\alpha_{i}=i / T$ and $\beta_{d_{i}}=\beta_{i}-1 / 2 T=i / T$, which proves the presence of information at $\beta_{d_{0}}=0$. Thus standard WL processing of $\mathrm{QR}$ signals, which corresponds to standard WL processing of $\mathbf{x}_{d}(t)$, exploits the information contained at $\left(\alpha_{d_{0}}, \beta_{d_{0}}\right)=$ $(0,0)$ through the exploitation of the temporal mean of the first correlation matrix, $\mathbf{R}_{\widetilde{x}_{d}}(t, \tau) \triangleq \mathrm{E}\left[\widetilde{\mathbf{x}}_{d}(t+\tau / 2) \widetilde{\mathbf{x}}_{d}^{H}(t-\tau / 2)\right]$, of the extended, or two-input, derotated model

$$
\widetilde{\mathbf{x}}_{d}(t) \triangleq\left[\mathbf{x}_{d}^{T}(t), \mathbf{x}_{d}^{H}(t)\right]^{T}=\sum_{k} b_{k} \widetilde{\mathbf{g}}_{d}(t-k T)+\widetilde{\mathbf{n}}_{d}(t),
$$

where $\widetilde{\mathbf{g}}_{d}(t) \triangleq\left[\mathbf{g}_{d}^{T}(t), \mathbf{g}_{d}^{H}(t)\right]^{T}$ and $\widetilde{\mathbf{n}}_{d}(t) \triangleq\left[\mathbf{n}_{d}^{T}(t), \mathbf{n}_{d}^{H}(t)\right]^{T}$. Comparing (11) and (15), we deduce that $\widetilde{\mathbf{x}}(t)$ for R signals 
and $\widetilde{\mathbf{x}}_{d}(t)$ for QR signals have similar forms, which explains why similar standard WL processing may be used for $\mathrm{R}$ and QR signals provided that the data vector $\mathbf{x}(t)$, used for $\mathrm{R}$ signals, is replaced by $\mathbf{x}_{d}(t)$ for $\mathrm{QR}$ signals. Due to the similarity of (11) and (15), it is implicitly accepted as true in the literature, that $\mathrm{R}$ and $\mathrm{QR}$ signals are equivalent, in terms of processing and performance, for standard WL filtering in the presence of CCI. We will show in section IV, for the first time to the best of our knowledge, that this commonly shared implicit assumption may not be true and that QR signals may be intrinsically less efficient than $\mathrm{R}$ ones for some standard WL filtering in the presence of CCI. The reasons explaining this result will be given in section $\mathrm{V}$ jointly with the way to make QR signals always almost equivalent to $\mathrm{R}$ ones for $\mathrm{WL}$ filtering in the presence of CCI.

\section{GENERIC PSEUDO-MLSE RECEIVER}

To compare $\mathrm{R}$ and $\mathrm{QR}$ signals for SAIC/MAIC from standard WL filtering, we need to introduce the receiver we have chosen, which corresponds here to a CT pseudo-MLSE receiver. Let us recall that the choice of a CT approach allows us to remove, both the filtering structure constraints generally imposed by a DT approach and the potential influence of the sample rate. Moreover, contrary to a DT approach, it allows us to obtain analytical interpretable performance computations at the output of all the receivers considered in this paper, which is completely original. On the other hand, the pseudo-MLSE approach is chosen here, since it is much easier to manipulate than an MLSE approach and it is generally much more powerful than an MMSE approach for frequency selective fading channels.

\section{A. Pseudo-MLSE approach}

In order to only exploit the information contained in the SO statistics of the data, and for both $\mathrm{R}$ and $\mathrm{QR}$ signals, the CT MLSE receiver for the detection of the symbols $b_{k}$, would assume a Gaussian total noise, despite the fact that the CCI are non-Gaussian R or QR signals. Note that the Gaussian assumption would nevertheless be approximately verified in practice in the presence of a high number of i.i.d. CCI. Moreover, to exploit the SO cyclostationarity and the SO non-circularity properties of the CCI, the total noise would be assumed to be SO cyclostationary and SO noncircular. However, under these assumptions, the CT MLSE receiver, which optimally exploits the CCI SO properties, is very challenging to derive, and even probably impossible to implement, at least for some pulse shaping filters $v(t)$. Such an MLSE receiver would optimally exploits the information contained in all the SO cyclic frequencies $\left(\alpha_{i}, \beta_{i}\right) i \in \mathbb{Z}$ of the total noise through the implementation of a potentially infinite number of time invariant (TI) filters acting on an infinite number of FRESH versions of $\mathbf{x}(t)$ and $\mathbf{x}^{*}(t)$, at least for some pulse shaping filters.

In this context, to overcome the difficulty to compute the CT MLSE receiver, a standard CT WL approach consists in only exploiting the non-circularity of the data, i.e. of $\mathbf{x}(t)$ and $\mathbf{x}_{d}(t)$ for $\mathrm{R}$ and $\mathrm{QR}$ signals, respectively, but not their cyclostationarity. In other words, it consists in computing the CT MLSE receiver from $\mathbf{x}(t)$ or $\mathbf{x}_{d}(t)$, for $\mathrm{R}$ and $\mathrm{QR}$ signals respectively, assuming a Gaussian non-circular but stationary total noise $\mathbf{n}(t)$ or $\mathbf{n}_{d}(t)$. It can be easily verified [53] that this approach is equivalent to computing the CT MLSE receiver from $\widetilde{\mathbf{x}}(t)$ (R signals) or $\widetilde{\mathbf{x}}_{d}(t)$ (QR signals) in Gaussian circular stationary extended total noise $\widetilde{\mathbf{n}}(t)$ or $\widetilde{\mathbf{n}}_{d}(t)$, respectively. To approximate the CT MLSE receiver in cyclostationary non-circular total noise, we adopt in the following the previous sub-optimal approach and we call it a CT two-input pseudo-MLSE approach. We will then compare in the following the output performance of the two-input pseudo-MLSE receivers computed from (11) and (15) for $\mathrm{R}$ and QR signals, respectively, corrupted by CCI of the same nature. Note that the conventional CT pseudo-MLSE receiver, called CT one-input pseudo MLSE receiver, corresponds to the CT MLSE receiver computed from $\mathbf{x}(t)$ (R signals) or $\mathbf{x}_{d}(t)$ (QR signals), assuming a Gaussian circular and stationary total noise $\mathbf{n}(t)$ or $\mathbf{n}_{d}(t)$, respectively.

\section{B. Generic pseudo-MLSE receiver}

For the $M$-input pseudo-MLSE receivers $(M=1,2)$, we denote by $\widetilde{\mathbf{x}}_{\mathrm{F}}(t)$ and $\widetilde{\mathbf{n}}_{\mathrm{F}}(t)$ the generic observation and total noise vectors, respectively. For conventional receivers $(M=1), \widetilde{\mathbf{x}}_{\mathrm{F}}(t)$ and $\widetilde{\mathbf{n}}_{\mathrm{F}}(t)$ reduce respectively to $\mathbf{x}(t)$ and $\mathbf{n}(t)$, for R signals, and to $\mathbf{x}_{d}(t)$ and $\mathbf{n}_{d}(t)$, for $\mathbf{Q R}$ signals. For $M=2$, these vectors correspond, for $\mathrm{R}$ signals, to $\widetilde{\mathbf{x}}(t)$ and $\widetilde{\mathbf{n}}(t)$, respectively, defined by (11), and for QR signals, to $\widetilde{\mathbf{x}}_{d}(t)$ and $\widetilde{\mathbf{n}}_{d}(t)$ respectively, defined by (15). Assuming a stationary, circular and Gaussian generic extended total noise $\widetilde{\mathbf{n}}_{\mathrm{F}}(t)$, it is shown in [53], [54] that the sequence $\widehat{\mathbf{b}} \triangleq\left(\widehat{b}_{1}, \ldots, \widehat{b}_{K}\right)$ which maximizes its likelihood from $\widetilde{\mathbf{x}}_{F}(t)$, is the one which minimizes the following criterion:

$$
C(\mathbf{b})=\int\left[\widetilde{\mathbf{x}}_{\mathrm{F}}(f)-\widetilde{\mathbf{s}}_{\mathrm{F}}(f)\right]^{H} \mathbf{R}_{\tilde{n}_{\mathrm{F}}}^{0}(f)^{-1}\left[\widetilde{\mathbf{x}}_{\mathrm{F}}(f)-\widetilde{\mathbf{s}}_{\mathrm{F}}(f)\right] d f .
$$

Here, $\mathbf{R}_{\tilde{n}_{F}}^{0}(f)$, the Fourier transform of $\mathbf{R}_{\tilde{n}_{F}}^{0}(\tau)$, corresponds to the power spectral density matrix of $\widetilde{\mathbf{n}}_{\mathrm{F}}(t)$. The signal $\widetilde{\mathbf{s}}_{\mathrm{F}}(f)$ is defined by $\widetilde{\mathbf{s}}_{\mathrm{F}}(f) \triangleq \sum_{k=1}^{K} b_{k} \widetilde{\mathbf{g}}_{\mathrm{F}}(f) e^{-j 2 \pi f k T}$, where $\widetilde{\mathbf{g}}_{\mathrm{F}}(f)$ corresponds, for $M=1$, to $\mathbf{g}(f)$ and $\mathbf{g}_{d}(f)$ for $\mathbf{R}$ and $\mathrm{QR}$ signals, respectively and for $M=2$ to $\widetilde{\mathbf{g}}(f)$ and $\widetilde{\mathbf{g}}_{d}(f)$ for $\mathrm{R}$ and $\mathrm{QR}$ signals, respectively. Considering only terms that depend on the symbols $b_{k}$, the minimization of (16) is equivalent to the minimization of the metric:

$$
\Lambda(\mathbf{b})=\sum_{k=1}^{K} \sum_{k^{\prime}=1}^{K} b_{k} b_{k^{\prime}} r_{k, k^{\prime}}-2 \sum_{k=1}^{K} b_{k} z_{\mathrm{F}}(k),
$$

where $z_{\mathrm{F}}(k)=\Re\left[y_{\mathrm{F}}(k)\right]$ and where $y_{\mathrm{F}}(k)$ and the coefficients $r_{k, k^{\prime}}$ are defined by

$$
\begin{aligned}
y_{\mathrm{F}}(k) & =\int \widetilde{\mathbf{g}}_{\mathrm{F}}^{H}(f)\left[\mathbf{R}_{\widetilde{n}_{\mathrm{F}}}^{0}(f)\right]^{-1} \widetilde{\mathbf{x}}_{\mathrm{F}}(f) e^{j 2 \pi f k T} d f, \\
r_{k, k^{\prime}} & =\int \widetilde{\mathbf{g}}_{\mathrm{F}}^{H}(f)\left[\mathbf{R}_{\widetilde{n}_{\mathrm{F}}}^{0}(f)\right]^{-1} \widetilde{\mathbf{g}}_{\mathrm{F}}(f) e^{j 2 \pi f\left(k-k^{\prime}\right) T} d f .
\end{aligned}
$$

Let us note that while $y_{\mathrm{F}}(k)$ is complex-valued for $M=1$, it becomes real-valued and corresponds to $z_{\mathrm{F}}(k)$ for $M=2$.

\section{Interpretation of the generic pseudo-MLSE receiver}

We deduce from (18) that $y_{\mathrm{F}}(k)$ is the sampled output, at time $t=k T$, of the TI filter whose frequency response is 


$$
\widetilde{\mathbf{w}}_{\mathrm{F}}^{H}(f) \triangleq\left(\left[\mathbf{R}_{\widetilde{n}_{\mathrm{F}}}^{0}(f)\right]^{-1} \widetilde{\mathbf{g}}_{\mathrm{F}}(f)\right)^{H},
$$

and whose input is $\widetilde{\mathbf{x}}_{\mathrm{F}}(t)$. The structure of the generic $M$-input pseudo-MLSE receiver $(M=1,2)$ is then depicted at Figure 1. It is composed of the TI WL filter (20), which reduces to a linear filter for $M=1$, followed by a sampling at the symbol rate, a real part capture (for $M=1$ ) and a decision box implementing the Viterbi algorithm, since $r_{k, k^{\prime}}^{*}=r_{k^{\prime}, k}$.

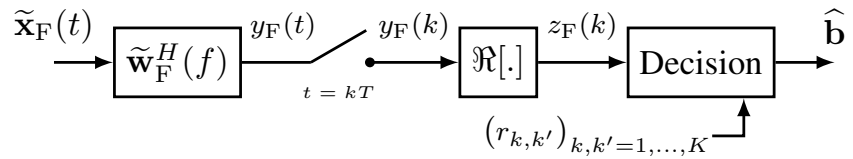

Fig. 1: Structure of the $M$-input $(M=1,2)$ pseudo-MLSE receiver

\section{Implementation of the generic pseudo-MLSE receiver}

The implementation of the generic $M$-input $(M=1,2)$ pseudo-MLSE receiver requires the knowledge or the estimation of $\widetilde{\mathbf{g}}_{\mathrm{F}}(f)$ and $\mathbf{R}_{\widetilde{n}_{\mathrm{F}}}^{0}(f)$ for each frequency $f$. This implementation is out of the scope of the paper but it requires the estimation of the channel impulse response of both the SOI and the CCI and the estimation of the background noise power spectral density.

\section{E. SINR at the output of the generic pseudo-MLSE receiver}

For real-valued symbols $b_{k}$, the SER at the output of the generic $M$-input $(M=1,2)$ pseudo-MLSE receiver is directly linked to the SINRs on the current symbol $n$ before decision, i.e. at the output $z_{\mathrm{F}}(n)$ [49, Sec 10.1.4], without taking into account the ISI which is processed by the decision box. For this reason, we compute the general expression of the output SINRs hereafter and we will analyze their variations for both $\mathrm{R}$ and QR signals in particular situations in section IV. As $\widetilde{\mathbf{n}}_{\mathrm{F}}(t)$ is SO cyclostationary and $\mathrm{SO}$ non-circular in the presence of CCI, the filter (20) does not maximize the output SINRs and can only be considered as a generic $M$-input pseudo-matched filter. It is easy to verify from (1), (11), (12), (15), (18) and (19), that $z_{\mathrm{F}}(n)$ can be written as

$$
z_{\mathrm{F}}(n)=b_{n} r_{n, n}+\sum_{k \neq n} b_{k} \Re\left[r_{n, k}\right]+z_{n, \mathrm{~F}}(n),
$$

where $z_{n, \mathrm{~F}}(n)=\Re\left[y_{n, \mathrm{~F}}(n)\right]$ and where $y_{n, \mathrm{~F}}(n)$ is defined by (18) for $k=n$ with $\widetilde{\mathbf{n}}_{\mathrm{F}}(f)$ instead of $\widetilde{\mathbf{x}}_{\mathrm{F}}(f)$. The output SINR on the current symbol $n$ is then defined by

$$
\operatorname{SINR}_{\mathrm{F}}(n) \triangleq \frac{\pi_{b} r_{n, n}^{2}}{\mathrm{E}\left[z_{n, \mathrm{~F}}^{2}(n)\right]}=\frac{2 \pi_{b} r_{n, n}^{2}}{\mathrm{E}\left[\left|y_{n, \mathrm{~F}}(n)\right|^{2}\right]+\Re\left(\mathrm{E}\left[y_{n, \mathrm{~F}}^{2}(n)\right]\right)} .
$$

In the presence of $\mathrm{R}$ or $\mathrm{QR} \mathrm{CCI}$, the total noise, $y_{n, \mathrm{~F}}(t)$, at the output of (20) is SO cyclostationary, which implies that $\mathrm{E}\left[\left|y_{n, \mathrm{~F}}(n)\right|^{2}\right]$ and $\mathrm{E}\left[y_{n, \mathrm{~F}}^{2}(n)\right]$ have Fourier series expansions given by [2]

$$
\begin{aligned}
\mathrm{E}\left[\left|y_{n, \mathrm{~F}}(n)\right|^{2}\right] & =\sum_{\gamma_{i}} e^{j 2 \pi \gamma_{i} n T} \int \mathrm{r}_{y_{n, \mathrm{~F}}}^{\gamma_{i}}(f) d f \\
\mathrm{E}\left[y_{n, \mathrm{~F}}^{2}(n)\right] & =\sum_{\delta_{i}} e^{j 2 \pi \delta_{i} n T} \int \mathrm{c}_{y_{n, \mathrm{~F}}}^{\delta_{i}}(f) d f .
\end{aligned}
$$

Here, the quantities $\gamma_{i}$ and $\delta_{i}$ denote the non-conjugate and conjugate SO cyclic frequencies of $y_{n, \mathrm{~F}}(t)$, respectively, whereas $\mathrm{r}_{y_{n, \mathrm{~F}}}^{\gamma_{i}}(f)$ and $\mathrm{c}_{y_{n, \mathrm{~F}}}^{\delta_{i}}(f)$ are the Fourier transforms of the first, $\mathrm{r}_{y_{n, \mathrm{~F}}}^{\gamma_{i}}(\tau)$, and second, $\mathrm{c}_{y_{n, \mathrm{~F}}}^{\delta_{i}}(\tau)$, cyclic correlation functions of $y_{n, \mathrm{~F}}(t)$ for the delay $\tau$ and the cyclic frequencies $\gamma_{i}$ and $\delta_{i}$ respectively. Moreover, as $y_{n, \mathrm{~F}}(t)$ is the output of the TI filter (20) whose input is $\widetilde{\mathbf{n}}_{\mathrm{F}}(t)$, we can write

$$
\begin{aligned}
\mathrm{r}_{y_{n, \mathrm{~F}}}^{\gamma_{i}}(f) & =\widetilde{\mathbf{w}}_{\mathrm{F}}^{H}\left(f+\frac{\gamma_{i}}{2}\right) \mathbf{R}_{\widetilde{n}_{\mathrm{F}}}^{\gamma_{i}}(f) \widetilde{\mathbf{w}}_{\mathrm{F}}\left(f-\frac{\gamma_{i}}{2}\right), \\
\mathrm{c}_{y_{n, \mathrm{~F}}}^{\delta_{i}}(f) & =\widetilde{\mathbf{w}}_{\mathrm{F}}^{H}\left(f+\frac{\delta_{i}}{2}\right) \mathbf{C}_{\widetilde{n}_{\mathrm{F}}}^{\delta_{i}}(f) \widetilde{\mathbf{w}}_{\mathrm{F}}^{*}\left(\frac{\delta_{i}}{2}-f\right),
\end{aligned}
$$

where $\mathbf{R}_{\tilde{n}_{\mathrm{F}}}^{\gamma_{i}}(f)$ and $\mathbf{C}_{\tilde{n}_{\mathrm{F}}}^{\delta_{i}}(f)$ are the Fourier transforms of the first, $\mathbf{R}_{\tilde{n}_{\mathrm{F}}}^{\gamma_{i}}(\tau)$, and second, $\mathbf{C}_{\tilde{n}_{\mathrm{F}}}^{\delta_{i}}(\tau)$ cyclic correlation matrices of $\widetilde{\mathbf{n}}_{\mathrm{F}}(t)$ for the delay $\tau$ and the cyclic frequencies $\gamma_{i}$ and $\delta_{i}$ respectively. Using (19) and (23) to (26) into (22), we obtain an alternative expression of (22) given by

$$
\begin{aligned}
& \operatorname{SINR}_{\mathrm{F}}(n)= \\
& \frac{2 \pi_{b}\left[\int \widetilde{\mathbf{g}}_{\mathrm{F}}^{H}(f) \mathbf{R}_{\widetilde{n}_{\mathrm{F}}}^{0}(f)^{-1} \widetilde{\mathbf{g}}_{\mathrm{F}}(f) d f\right]^{2}}{\left\{\begin{array}{l}
\sum_{\gamma_{i}} e^{j 2 \pi \gamma_{i} n T} \int \widetilde{\mathbf{w}}_{\mathrm{F}}^{H}\left(f+\frac{\gamma_{i}}{2}\right) \mathbf{R}_{\tilde{n}_{\mathrm{F}}}^{\gamma_{i}}(f) \widetilde{\mathbf{w}}_{\mathrm{F}}\left(f-\frac{\gamma_{i}}{2}\right) d f \\
+\Re\left(\sum_{\delta_{i}} e^{j 2 \pi \delta_{i} n T} \int \widetilde{\mathbf{w}}_{\mathrm{F}}^{H}\left(f+\frac{\delta_{i}}{2}\right) \mathbf{C}_{\tilde{n}_{\mathrm{F}}}^{\delta_{i}}(f) \mathbf{w}_{\mathrm{F}}^{*}\left(\frac{\delta_{i}}{2}-f\right) d f\right)
\end{array}\right.} .
\end{aligned}
$$

In the presence of $\mathrm{CCI}$ having same nature (R or $\mathrm{QR}$ ), symbol period and carrier frequency as the SOI, for $M=1,2$ and for both $\mathrm{R}$ and $\mathrm{QR}$ signals, the non-conjugate $\gamma_{i}$ and conjugate $\delta_{i}$ SO cyclic frequencies of the output $y_{n, \mathrm{~F}}(t)$ of the filter $\widetilde{\mathbf{w}}_{\mathrm{F}}(f)$ are those of the input $\widetilde{\mathbf{n}}_{\mathrm{F}}(t)$, which are from (13) and (14) $\gamma_{i}=\delta_{i}=\alpha_{i}=i / T, i \in \mathbb{Z}$. This implies that $\operatorname{SINR}_{\mathrm{F}}(n)$ given by (27) does not depend on $n$ and is simply denoted by SINR $_{\mathrm{F}}$, whose expression is given by

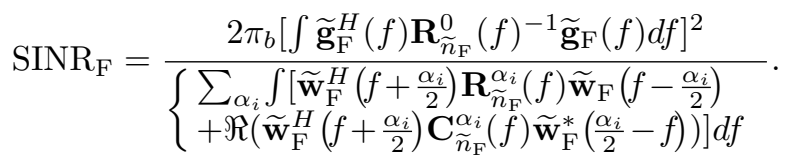

As $y_{n, \mathrm{~F}}(n)$ is real-valued for the extended models (11) and (15), $\mathrm{SINR}_{\mathrm{F}}$ reduces, for $M=2$, to

$$
\operatorname{SINR}_{\mathrm{F}}=\frac{\pi_{b}\left[\int \widetilde{\mathbf{g}}_{\mathrm{F}}^{H}(f) \mathbf{R}_{\widetilde{n}_{\mathrm{F}}}^{0}(f)^{-1} \widetilde{\mathbf{g}}_{\mathrm{F}}(f) d f\right]^{2}}{\sum_{\alpha_{i}} \int \widetilde{\mathbf{w}}_{\mathrm{F}}^{H}\left(f+\frac{\alpha_{i}}{2}\right) \mathbf{R}_{\widetilde{n}_{\mathrm{F}}}^{\alpha_{i}}(f) \widetilde{\mathbf{w}}_{\mathrm{F}}\left(f-\frac{\alpha_{i}}{2}\right) d f} ;(M=2) .
$$

\section{SINR ANALYSIS FOR ONE CCI}

\section{A. Total noise model and statistics}

We assume in this section IV that the total noise $\mathbf{n}(t)$ is composed of a background noise and one multi-user CCI, having the same nature, symbol period and carrier frequency as the SOI. In this context, the first purpose of this section is to verify, for both $\mathrm{R}$ and $\mathrm{QR}$ signals, the effectiveness of the two-input pseudo-MLSE receiver with respect to the conventional one for CCI mitigation for most of frequency selective propagation channels, even for $N=1$. The second purpose of this section is then to prove the lower efficiency of the two-input pseudo-MLSE receiver for QR signals with respect to $\mathrm{R}$ ones. Under the previous assumption, $\mathbf{n}(t)$ can be written as

$$
\mathbf{n}(t)=\sum_{k} c_{k} \mathbf{g}_{I}(t-k T)+\mathbf{u}(t) .
$$


Here, $c_{k}=d_{k}$ for $\mathrm{R}$ signals whereas $c_{k}=j^{k} d_{k}$ for $\mathrm{QR}$ signals, where $d_{k}$ are real-valued zero-mean i.i.d. random variables, corresponding to the CCI symbols for an R interference and directly related to the CCI symbols for a $\mathrm{QR}$ interference, $\mathbf{g}_{I}(t) \triangleq v(t) \otimes \mathbf{h}_{I}(t), \mathbf{h}_{I}(t)$ is the $N \times 1$ impulse response of the propagation channel of the CCI and $\mathbf{u}(t)$ is the $N \times 1$ background noise vector, assumed stationary, temporally and spatially white. From (30), it is proved in Appendix A, that for both a R and a QR CCI and for $M=1,2$, the matrices $\mathbf{R}_{\tilde{n}_{\mathrm{F}}}^{\alpha_{i}}(f)$ and $\mathbf{C}_{\tilde{n}_{\mathrm{F}}}^{\alpha_{i}}(f)$ appearing in (28) can be written as

$$
\begin{array}{r}
\mathbf{R}_{\tilde{n}_{\mathrm{F}}}^{\alpha_{i}}(f)=\frac{\pi_{d}}{T} \widetilde{\mathbf{g}}_{I_{\mathrm{F}}}\left(f+\frac{\alpha_{i}}{2}\right) \widetilde{\mathbf{g}}_{I_{\mathrm{F}}}^{H}\left(f-\frac{\alpha_{i}}{2}\right)+N_{0} \delta\left(\alpha_{i}\right) \mathbf{I}_{M N}, \\
\mathbf{C}_{\widetilde{n}_{\mathrm{F}}}^{\alpha_{i}}(f)=\frac{\pi_{d}}{T} \widetilde{\mathbf{g}}_{I_{\mathrm{F}}}\left(f+\frac{\alpha_{i}}{2}\right) \widetilde{\mathbf{g}}_{I_{\mathrm{F}}}^{T}\left(\frac{\alpha_{i}}{2}-f\right)+N_{0} \delta\left(\alpha_{i}\right) \delta(M-2) \mathbf{J}_{2 N} .
\end{array}
$$

Here $\pi_{d} \triangleq \mathrm{E}\left[d_{k}^{2}\right], N_{0}$ is the power spectral density of each component of the background noise $\mathbf{u}(t), \widetilde{\mathbf{g}}_{I_{\mathrm{F}}}(f)$ is defined as $\widetilde{\mathbf{g}}_{\mathrm{F}}(f)$ but with $\mathbf{g}_{I}(f)$ instead of $\mathbf{g}(f)$ and $\mathbf{J}_{2 N}$ is the $(2 N \times$ $2 N$ ) matrix defined by

$$
\mathbf{J}_{2 N} \triangleq\left[\begin{array}{ll}
\mathbf{0}_{N} & \mathbf{I}_{N} \\
\mathbf{I}_{N} & \mathbf{0}_{N}
\end{array}\right]
$$

\section{B. SINR computation and analysis for $M=2$ and a strong} interference

Let us assume in this section that $M=2$ and let us define the quantity $\widetilde{\varepsilon}_{I_{\mathrm{F}}}(f)$ by

$$
\widetilde{\varepsilon}_{I_{\mathrm{F}}}(f) \triangleq \frac{\pi_{d}}{N_{0} T} \widetilde{\mathbf{g}}_{I_{\mathrm{F}}}^{H}(f) \widetilde{\mathbf{g}}_{I_{\mathrm{F}}}(f) .
$$

We denote by $B_{\mathrm{F}}^{0}$ the set of frequencies $f$ such that $\widetilde{\mathbf{g}}_{\mathrm{F}}(f)$ is non-zero. Assuming a strong CCI for which $\widetilde{\varepsilon}_{I_{\mathrm{F}}}(f) \gg 1$ when $\widetilde{\varepsilon}_{I_{\mathrm{F}}}(f) \neq 0$ for $f \in B_{\mathrm{F}}^{0}$, it is proved in Appendix B that $\mathrm{SINR}_{\mathrm{F}}$ can be approximated, for both R and QR strong CCI, by

$$
\operatorname{SINR}_{\mathrm{F}} \approx \frac{\pi_{b}}{N_{0}} \int_{B_{\mathrm{F}}^{0}} \widetilde{\mathbf{g}}_{\mathrm{F}}^{H}(f) \widetilde{\mathbf{g}}_{\mathrm{F}}(f)\left[1-\left|\widetilde{\alpha}_{S I_{\mathrm{F}}}(f)\right|^{2}\right] d f,
$$

as long as $\operatorname{SINR}_{\mathrm{F}}$ is non-zero. Here, $\widetilde{\alpha}_{S I_{\mathrm{F}}}(f)$, such that $0 \leq$ $\left|\widetilde{\alpha}_{S I_{\mathrm{F}}}(f)\right| \leq 1$, is the extended spatial correlation coefficient between the SOI and the CCI for the frequency $f$ and the observation model $\widetilde{\mathbf{x}}_{\mathrm{F}}(t)$, defined by

$$
\widetilde{\alpha}_{S I_{\mathrm{F}}}(f) \triangleq \frac{\widetilde{\mathbf{g}}_{\mathrm{F}}^{H}(f) \widetilde{\mathbf{g}}_{I_{\mathrm{F}}}(f)}{\sqrt{\widetilde{\mathbf{g}}_{\mathrm{F}}^{H}(f) \widetilde{\mathbf{g}}_{\mathrm{F}}(f)} \sqrt{\widetilde{\mathbf{g}}_{I_{\mathrm{F}}}^{H}(f) \widetilde{\mathbf{g}}_{I_{\mathrm{F}}}(f)}} .
$$

For $N=1$, a receiver performs SAIC as the CCI becomes infinitely strong if the associated $\mathrm{SINR}_{\mathrm{F}}$ does not converge toward zero. Expression (35) then shows that for $M=2$ and for both R and QR signals, the WL filter (20) performs SAIC for SOI and CCI propagation channels such that $\left|\widetilde{\alpha}_{I S_{\mathrm{F}}}(f)\right|$ is not constant and equal to 1 over $B_{\mathrm{F}}^{0}$, i.e. for most of propagation channels. This result, which, to the best of our knowledge, has never been published in the literature, enlightens, for both $\mathrm{R}$ and $\mathrm{QR}$ signals, the interest and the effectiveness of the associated two-input pseudo-MLSE receivers for most of frequency selective SOI and CCI propagation channels.
C. SINR computation and analysis for $M=1,2$ and channels with no delay spread

1) Propagation channel model: To get more insights into the comparative behavior of the $M$-input pseudo-MLSE receivers $(M=1,2)$ for $\mathrm{R}$ and $\mathrm{QR}$ signals, we assume in this section IV-C a square root raised cosine (SRRC) pulse shaping filter ( $1 / 2$ Nyquist filter) $v(t)$ with a roll off $\omega$ and, to simplify the analysis and the analytical computations, propagation channels with no delay spread such that

$$
\mathbf{h}(t)=\mu \delta(t) \mathbf{h} \quad \text { and } \quad \mathbf{h}_{I}(t)=\mu_{I} \delta\left(t-\tau_{I}\right) \mathbf{h}_{I} .
$$

Here, $\mu$ and $\mu_{I}$ control the amplitude of the SOI and CCI respectively and $\tau_{I}$ is the delay of the CCI with respect to the SOI. The vectors $\mathbf{h}$ and $\mathbf{h}_{I}$, random or deterministic, with components $h(i)$ and $h_{I}(i)(1 \leq i \leq N)$, respectively and such that $\mathrm{E}\left[|h(i)|^{2}\right]=\mathrm{E}\left[\left|h_{I}(i)\right|^{2}\right]=1(1 \leq i \leq N)$, correspond to the channel vectors of the SOI and CCI, respectively. The mean powers of the SOI and CCI at the output of each antenna are given by $P_{s} \triangleq\left\langle\mathrm{E}\left[|\mu s(t) h(i)|^{2}\right]\right\rangle=\mu^{2} \pi_{b} / T$ and $P_{j} \triangleq\left\langle\mathrm{E}\left[\left|\mu_{I} j(t) h_{I}(i)\right|^{2}\right]\right\rangle=\mu_{I}^{2} \pi_{d} / T$ respectively, where $j(t)$ is defined by (8) with $c_{k}$ instead of $a_{k}$.

2) Deterministic channels and zero roll-off: Under the previous assumptions, analytical interpretable expressions of SINR $_{F}$ defined by (28) are only possible for a zero rolloff $\omega$, which is assumed in this sub-section. Otherwise, the computation of (28) can only be done numerically by computer simulations and will be discussed in the following sub-section. For a zero roll-off, the quantities $\pi_{s} \triangleq \mu^{2} \pi_{b}$, $\pi_{I} \triangleq \mu_{I}^{2} \pi_{d}$ and $N_{0}$ correspond to the mean power of the $\mathrm{SOI}$, the CCI and the background noise per antenna at the output of the pulse shaping matched filter respectively. We then denote by $\varepsilon_{s}$ and $\varepsilon_{I}$ the quantities $\varepsilon_{s} \triangleq \pi_{s} \mathbf{h}^{H} \mathbf{h} / N_{0}$ and $\varepsilon_{I} \triangleq \pi_{I} \mathbf{h}_{I}^{H} \mathbf{h}_{I} / N_{0}$ and by $\operatorname{SINR}_{\mathrm{R}_{M}}$ and $\operatorname{SINR}_{\mathrm{QR}_{M}}$ the SINR (28) at the output of the $M$-input pseudo-MLSE receiver for $\mathrm{R}$ and $\mathrm{QR}$ signals respectively. Moreover, we assume in this sub-section deterministic channels and we denote by $\alpha_{s I}$ the spatial correlation coefficient between the SOI and the CCI, such that $\left(0 \leq\left|\alpha_{s I}\right| \leq 1\right)$, and defined by

$$
\alpha_{s I} \triangleq \frac{\mathbf{h}^{H} \mathbf{h}_{I}}{\sqrt{\mathbf{h}^{H} \mathbf{h}} \sqrt{\mathbf{h}_{I}^{H} \mathbf{h}_{I}}} \triangleq\left|\alpha_{s I}\right| e^{j \phi_{s I}} .
$$

Note that (36) reduces to (38) for $M=1$ and propagation channels (37).

When $\left|\alpha_{s I}\right| \neq 1$, i.e. when there exists a spatial discrimination between the SOI and the CCI (which requires $N>1$ ), assuming a strong CCI $\left(\varepsilon_{I} \gg 1\right)$, we obtain from (20), (28), (31), (32), (37), (38), and after straightforward derivations, the following expressions:

$$
\begin{aligned}
\operatorname{SINR}_{\mathrm{R}_{1}} & \approx \operatorname{SINR}_{\mathrm{QR}_{1}} \approx 2 \varepsilon_{s}\left[1-\left|\alpha_{s I}\right|^{2}\right], \\
\operatorname{SINR}_{\mathrm{R}_{2}} & \approx 2 \varepsilon_{s}\left[1-\left|\alpha_{s I}\right|^{2} \cos ^{2}\left(\phi_{s I}\right)\right], \\
\operatorname{SINR}_{\mathrm{QR}_{2}} & \approx 2 \varepsilon_{s}\left[1-\frac{\left|\alpha_{s I}\right|^{2}}{2}\left\{1+\cos ^{2}\left(\psi_{s I}\right)\right\}\right],
\end{aligned}
$$

where $\psi_{s I} \triangleq \phi_{s I}-\pi \tau_{I} / 2 T$. However, when $\left|\alpha_{s I}\right|=1$, i.e. when there is no spatial discrimination between the SOI and 
the CCI, which is in particular the case for $N=1$, after simple computations, $\mathrm{SINR}_{\mathrm{R}_{1}}$ and $\mathrm{SINR}_{\mathrm{QR}_{1}}$ can be written as

$$
\begin{aligned}
\operatorname{SINR}_{\mathrm{R}_{1}} & =\frac{2 \varepsilon_{s}}{1+2 \varepsilon_{I} \cos ^{2}\left(\phi_{s I}\right)}, \\
\mathrm{SINR}_{\mathrm{QR}_{1}} & =\frac{2 \varepsilon_{s}}{\left.1+\varepsilon_{I}\left[1-\cos \left(\frac{\pi \tau_{I}}{T}\right)+2 \cos \left(\frac{\pi \tau_{I}}{T}\right) \cos ^{2}\left(\phi_{s I}\right)\right)\right]},
\end{aligned}
$$

whereas, assuming a strong CCI $\left(\varepsilon_{I} \gg 1\right), \operatorname{SINR}_{\mathrm{R}_{2}}$ and $\mathrm{SINR}_{\mathrm{QR}_{2}}$ can be written as

$$
\begin{aligned}
\operatorname{SINR}_{\mathrm{R}_{2}} \approx 2 \varepsilon_{s}\left[1-\cos ^{2}\left(\phi_{s I}\right)\right], & & \phi_{s I} \neq k \pi, \\
\operatorname{SINR}_{\mathrm{QR}_{2}} \approx 2 \varepsilon_{s}\left[1-\frac{1+\cos ^{2}\left(\psi_{s I}\right)}{2}\right], & & \psi_{s I} \neq k \pi .
\end{aligned}
$$

Finally, for R signals such that $\left|\alpha_{s I}\right|=1$ and $\phi_{s I}=k \pi$ and for QR signals such that $\left|\alpha_{s I}\right|=1$ and $\psi_{s I}=k \pi$, we obtain

$$
\begin{aligned}
\operatorname{SINR}_{\mathrm{R}_{2}} & =\frac{2 \varepsilon_{s}}{1+2 \varepsilon_{I}}, & \phi_{s I} & =k \pi, \\
\mathrm{SINR}_{\mathrm{QR}_{2}} & =\frac{9 \varepsilon_{s}}{2 \varepsilon_{I}\left[3+2 \cos \left(4 \phi_{s I}\right)\right]}, & \psi_{s I} & =k \pi .
\end{aligned}
$$

Note that (39), (40), (42), (44) and (46), i.e. output SINR for $\mathrm{R}$ signals, have been obtained in [8] but from a DT MMSE approach. However, concerning the output SINR of QR signals, (45) and (47) have been given in [37], [38] but without any proof, whereas (41) and (43) have never been presented and are completely new. A receiver performs MAIC (for $N>1$ ) or SAIC (for $N=1$ ) as $\varepsilon_{I} \rightarrow \infty$, if the associated SINR does not converge toward zero. We deduce from (39), (42) and (43) that, for both R and QR signals, the conventional receivers perform MAIC as soon as $\left|\alpha_{s I}\right| \neq 1$, but perform SAIC very scarcely, only when $\phi_{s I}=(2 k+1) \pi / 2$ for $\mathrm{R}$ signals and when $\left(\tau_{I} / T, \phi_{s I}\right)=\left(2 k_{1},\left(2 k_{2}+1\right) \pi / 2\right)$ or $\left(2 k_{1}+1, k_{2} \pi\right)$ for QR signals, where $k, k_{1}$ and $k_{2}$ are integers. Moreover, we deduce from (40), (41), (44) and (45) that, for both R and QR signals, the two-input pseudo-MLSE receivers perform MAIC as soon as $\left|\alpha_{s I}\right| \neq 1$, but perform SAIC as long as $\phi_{s I} \neq k \pi$ for $\mathrm{R}$ signals and $\psi_{s I} \neq k \pi$ for QR signals, enlightening the great interest of the twoinput WL filtering (20) in both cases. However, despite similar processing (20) and similar extended models (11) and (15) for $\mathrm{R}$ and $\mathrm{QR}$ signals respectively, the output SINRs (40) and (41), for $\left|\alpha_{s I}\right| \neq 1$, and (44) and (45), for $\left|\alpha_{s I}\right|=1$, correspond to different expressions. This proves the non equivalence of $\mathrm{R}$ and derotated QR signals for the efficient WL filtering (20) in the presence of CCI, result which may be surprising for most of researchers on WL filtering. In particular, for a zero rolloff $\omega$, while (40) only depends on $2 \varepsilon_{s}$, the maximum output SINR obtained without interference, and the parameters $\alpha_{s I}$ and $\phi_{s I}$, (41) depends not only on the previous parameters but also on $\tau_{I} / T$.

Figure 2 and 3 show the variations of $\operatorname{SINR}_{\mathrm{R}_{M}}$ and $\operatorname{SINR}_{\mathrm{QR}_{M}}(M=1,2)$ as a function of $\phi_{s I}$ for $N=1$, $\varepsilon_{s}=10 \mathrm{~dB}, \varepsilon_{I}=20 \mathrm{~dB}$ for synchronous $\left(\tau_{I}=0\right)$ and asynchronous $\left(\tau_{I}=T\right)$ SOI and CCI, respectively. Let us note that for $\tau_{I}=T$, the curves related to $\mathrm{QR}$ signals are simply shifted of $-\pi / 2$ with respect to those obtained for $\tau_{I}=0$. Contrary to the conventional receiver, we note a SAIC capability of the two-input pseudo-MLSE receiver for both $\mathrm{R}$ and $\mathrm{QR}$ signals as soon as there is a phase discrimination between the sources. For $\tau_{I}=0$, we note better performance obtained for $\mathrm{R}$ signals with respect to $\mathrm{QR}$ signals and, for QR signals, the surprising better performance obtained with a 1-input instead of a 2-input pseudo-MLSE receiver for the very particular case $\phi_{s I}=\pi / 2$. This surprising result for this very specific case is nothing else than an artefact due to the sub-optimality of the pseudo-MLSE approach for a SO cyclostationary and non-circular total noise. For $\tau_{I}=T$, the same very specific artefact holds for $\phi_{s I}=0$ (i.e. for $\psi_{s I}=\pi / 2$ ) for the same reasons and the performance obtained for QR signals may be either better or worse than those obtained with $\mathrm{R}$ signals, depending on the value of $\phi_{s I}$.

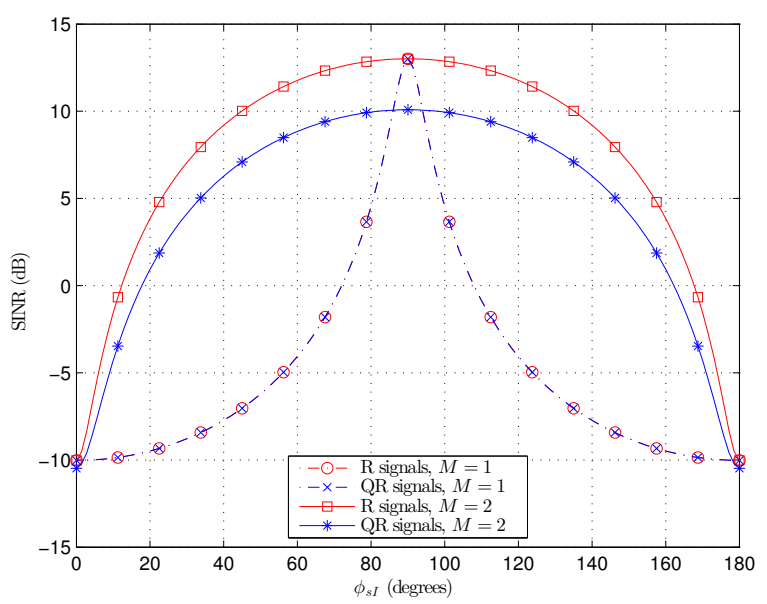

Fig. 2: $\operatorname{SINR}_{\mathrm{R}_{M}}$ and $\operatorname{SINR}_{\mathrm{QR}_{M}}(M=1,2)$ as a function of $\phi_{s I}\left(N=1, \tau_{I}=0, \varepsilon_{s}=10 \mathrm{~dB}, \varepsilon_{I}=20 \mathrm{~dB}\right.$, deterministic one tap channels)

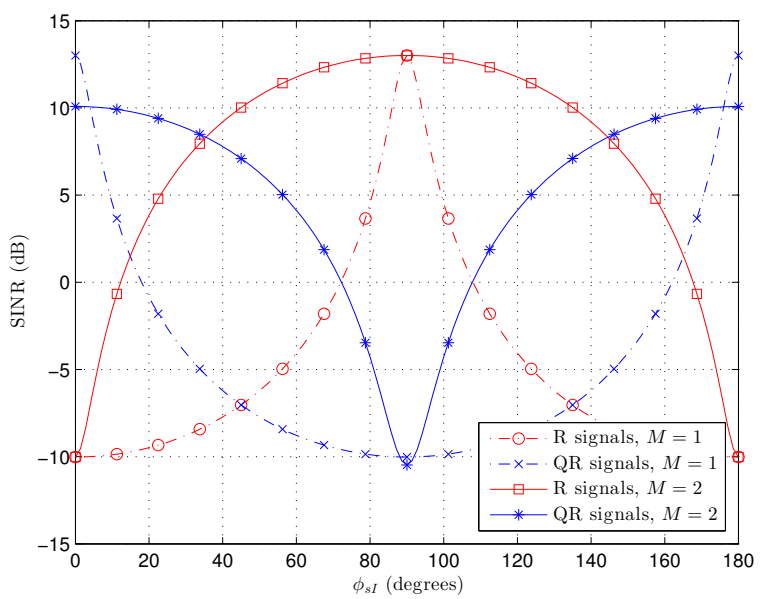

Fig. 3: $\operatorname{SINR}_{\mathrm{R}_{M}}$ and $\operatorname{SINR}_{\mathrm{QR}_{M}}(M=1,2)$ as a function of $\phi_{s I}\left(N=1, \tau_{I}=T, \varepsilon_{s}=10 \mathrm{~dB}, \varepsilon_{I}=20 \mathrm{~dB}\right.$, deterministic one tap channels)

For this reason, to compare $\mathrm{SINR}_{\mathrm{R}_{2}}$ and $\mathrm{SINR}_{\mathrm{QR}_{2}}$ for $\omega=0$ and $\varepsilon_{I} \gg 1$ whatever the value of $\tau_{I}$, we must adopt a statistical perspective. Consequently, we now assume that $\varepsilon_{I} \rightarrow \infty$ and $\phi_{s I}$ and $\pi \tau_{I} / 2 T$ are independent random variables uniformly distributed on $[0,2 \pi]$. Under these assumptions, we easily deduce from (40), (41), (44) and (45) the 
expectation value of the output SINRs given by

$$
\begin{aligned}
\mathrm{E}\left[\mathrm{SINR}_{\mathrm{R}_{2}}\right] & \approx 2 \varepsilon_{s}\left[1-\frac{\left|\alpha_{s I}\right|^{2}}{2}\right], \\
\mathrm{E}\left[\mathrm{SINR}_{\mathrm{QR}_{2}}\right] & \approx 2 \varepsilon_{s}\left[1-\frac{3\left|\alpha_{s I}\right|^{2}}{4}\right],
\end{aligned}
$$

which reduce, for $N=1$, to

$$
\mathrm{E}\left[\mathrm{SINR}_{\mathrm{R}_{2}}\right] \approx \varepsilon_{s} \quad \text { and } \mathrm{E}\left[\mathrm{SINR}_{\mathrm{QR}_{2}}\right] \approx \frac{\varepsilon_{s}}{2} .
$$

We clearly observe that $\mathrm{E}\left[\mathrm{SINR}_{\mathrm{QR}_{2}}\right]<\mathrm{E}\left[\mathrm{SINR}_{\mathrm{R}_{2}}\right]$ for $\left|\alpha_{s I}\right| \neq 0$, which definitely proves, at least for a zero rolloff, that $\mathrm{QR}$ signals are less efficient that $\mathrm{R}$ ones for the WL receiver (20) in the presence of one CCI, result which is unknown by most of the researchers. As, for $\tau_{I} \neq 0$, the curve showing the variations of $\mathrm{SINR}_{\mathrm{QR}_{2}}$ as a function of $\phi_{S I}$ is a shifted version, by the value $-\pi \tau_{I} / 2 T$, of the same curve for $\tau_{I}=0, \mathrm{E}\left[\mathrm{SINR}_{\mathrm{QR}_{2}}\right]$ would be the same as (49) and (50) for a fixed value of $\tau_{I}$, assuming that $\phi_{S I}$ is uniformly distributed on $[0,2 \pi]$. This proves that the delay $\tau_{I}$ does not impact the average value of $\mathrm{SINR}_{\mathrm{QR}_{2}}$.

3) Deterministic channels and arbitrary roll-off: To extend the previous results for arbitrary values of the roll-off $\omega$, we still assume that $\phi_{s I}$ and $\pi \tau_{I} / 2 T$ are independent random variables uniformly distributed on $[0,2 \pi]$. Under these assumptions, choosing $\varepsilon_{s}=10 \mathrm{~dB}$ and $\varepsilon_{I}=20 \mathrm{~dB}$, Figure 4 shows, for R and QR signals, for $N=1, M=1,2$ and $\omega=0,0.5$, the variations of $\operatorname{Pr}\left[\left(\mathrm{SINR}_{\mathrm{F}} / 2 \varepsilon_{s}\right) \mathrm{dB} \geq x \mathrm{~dB}\right] \triangleq \mathcal{P}_{\mathrm{F}}(x)$ as a function of $x(\mathrm{~dB})$. Note that the curves appearing in this Figure are obtained from Monte-Carlo simulations where SINR $_{F}$ has been computed from the general expressions (28) and (29).

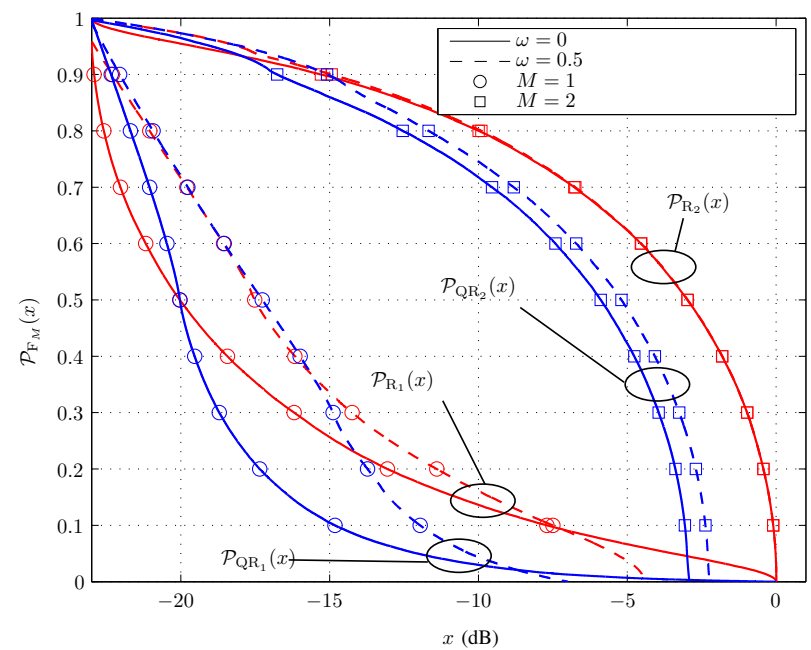

Fig. 4: $\mathcal{P}_{\mathrm{F}_{M}}(x)$ as a function of $x\left(N=1, \varepsilon_{s}=10 \mathrm{~dB}\right.$, $\varepsilon_{I}=20 \mathrm{~dB}, \omega=0,0.5$, deterministic one-tap channels, $\mathrm{R}$ and $\mathrm{QR}$ signals)

Note, for both $\mathrm{R}$ and $\mathrm{QR}$ signals, poor performance whatever $\omega$ for $M=1$, i.e. for the conventional receiver. Note for $M=2$, increasing and constant performance with $\omega$ for $\mathrm{QR}$ and $\mathrm{R}$ signals respectively, and the best performance of the receiver implemented from (11) with respect to (15) whatever $\omega$. This confirms, for arbitrary values of $\omega$, the lowest efficiency of QR signals with respect to R ones for SAIC from the two-input pseudo MLSE receiver. Note in particular, for $\omega=0.5$ and $x=-3 \mathrm{~dB}$, that $\mathcal{P}_{\mathrm{QR}_{1}}(x)=\mathcal{P}_{\mathrm{R}_{1}}(x)=0 \%$, $\mathcal{P}_{\mathrm{QR}_{2}}(x)=26 \%$ and $\mathcal{P}_{\mathrm{R}_{2}}(x)=50 \%$, proving the much better performance of the receiver implemented from (11) with respect to (15).

\section{ENHANCED SAIC/MAIC RECEIVER FOR QR SIGNALS}

We describe in this section the reasons why QR signals may be less efficient than R ones for standard WL filtering in the presence of $\mathrm{CCI}$ and we propose, for QR signals, a WL filtering enhancement to make them always almost equivalent to R signals.

\section{A. The lower efficiency of $Q R$ signals}

The lower efficiency of QR signals with respect to $\mathrm{R}$ ones for SAIC/MAIC from the two-input pseudo-MLSE receiver is directly related to the different $\mathrm{SO}$ non-circularity and $\mathrm{SO}$ cyclostationarity properties of $\mathrm{QR}$ and $\mathrm{R}$ signals. Indeed, the main information about the SO non-circularity of $\mathrm{R}$ signals is contained in the conjugate SO cyclic frequency $\beta_{0}=0$ whatever the real-valued filter $v(t)$, and this is all the more true as the filter roll-off $\omega$ decreases. As the two-input pseudoMLSE receiver applied to the model (11) exploits the information contained in $\left(\alpha_{0}, \beta_{0}\right)=(0,0)$, it always exploits most of the SO non-circularity information of $\mathrm{R}$ signals, hence its very good performance. On the contrary, the main information about the SO non-circularity of QR signals is always symmetrically contained in the two conjugate $\mathrm{SO}$ cyclic frequencies $\left(\beta_{0}, \beta_{-1}\right)=(1 / 2 T,-1 / 2 T)$, as illustrated in [51], [52], or equivalently in $\left(\beta_{d_{0}}, \beta_{d_{-1}}\right)=(0,-1 / T)$ for derotated $\mathrm{QR}$ signals. As a consequence, as the two-input pseudo-MLSE receiver applied to the model (15) exploits the information contained in $\left(\alpha_{d_{0}}, \beta_{d_{0}}\right)=(0,0)$, or in $\left(\alpha_{0}, \beta_{0}\right)=(0,1 / 2 T)$, it only exploits half of the main SO non-circularity information of QR signals, hence its sub-optimality.

\section{B. Three-input FRESH model}

To overcome, for QR signals, the limitations of the twoinput pseudo-MLSE receiver implemented from model (15), it is necessary to implement a WL receiver which is able to take full account of the main SO non-circularity information of QR signals. Such a receiver can be obtained by implementing the pseudo-MLSE receiver from the three-input FRESH model defined by

$$
\begin{aligned}
\mathbf{x}_{\mathrm{F}_{3}}(t) & \triangleq\left[\mathbf{x}^{T}(t), e^{j 2 \pi t / 2 T} \mathbf{x}^{H}(t), e^{-j 2 \pi t / 2 T} \mathbf{x}^{H}(t)\right]^{T} \\
& =j^{t / T}\left[\widetilde{\mathbf{x}}_{d}^{T}(t), e^{-j 2 \pi t / T} \mathbf{x}_{d}^{H}(t)\right]^{T} \triangleq j^{t / T} \mathbf{x}_{d \mathrm{~F}_{3}}(t) \\
& =\sum_{k} j^{k} b_{k} \mathbf{g}_{\mathrm{F}_{3}}(t-k T)+\mathbf{n}_{\mathrm{F}_{3}}(t),
\end{aligned}
$$

or equivalently from $\mathbf{x}_{d \mathrm{~F}_{3}}(t)$. Here, $\mathbf{n}_{\mathrm{F}_{3}}(t)$ corresponds to $\mathbf{x}_{\mathrm{F}_{3}}(t)$ with $\mathbf{n}(t)$ instead of $\mathbf{x}(t)$ whereas $\mathbf{g}_{\mathrm{F}_{3}}(t) \triangleq$ $\left[\mathbf{g}^{T}(t), e^{j 2 \pi t / 2 T} \mathbf{g}^{H}(t), e^{-j 2 \pi t / 2 T} \mathbf{g}^{H}(t)\right]^{T}$. It is straightforward to verify that the temporal mean of the first correlation matrices, $\mathbf{R}_{x_{\mathrm{F}_{3}}}(t, \tau) \triangleq \mathrm{E}\left[\mathbf{x}_{\mathrm{F}_{3}}(t+\tau / 2) \mathbf{x}_{\mathrm{F}_{3}}^{H}(t-\tau / 2)\right]$ and $\mathbf{R}_{x_{d \mathrm{~F}_{3}}}(t, \tau) \triangleq \mathrm{E}\left[\mathbf{x}_{d \mathrm{~F}_{3}}(t+\tau / 2) \mathbf{x}_{d \mathrm{~F}_{3}}^{H}(t-\tau / 2)\right]$, of $\mathbf{x}_{\mathrm{F}_{3}}(t)$ and $\mathbf{x}_{d \mathrm{~F}_{3}}(t)$ respectively exploit the information contained in $\left(\alpha_{0}, \alpha_{-1}, \alpha_{1}, \beta_{0}, \beta_{-1}\right)=(0,-1 / T, 1 / T, 1 / 2 T,-1 / 2 T)$, 
which allows us to exploit almost exhaustively both the SO cyclostationarity and the SO non-circularity properties of QR signals. Note that a TI linear processing of $\mathbf{x}_{\mathrm{F}_{3}}(t)$ (resp. $\mathbf{x}_{d \mathrm{~F}_{3}}(t)$ ) becomes now a time variant (TV) WL processing of $\mathbf{x}(t)$ (resp. $\mathbf{x}_{d}(t)$ ), called here three-input WL FRESH processing of $\mathbf{x}(t)$ (resp. $\left.\mathbf{x}_{d}(t)\right)$. Note finally that, since model (51) allows us to take into account, at least for SRRC filters, the main information about SO cyclostationarity and SO noncircularity of $\mathrm{QR}$ signals, there is little interest to consider $M$-input FRESH models with $M>3$ for SAIC/MAIC.

\section{Three-input pseudo-MLSE receiver}

Applying the generic pseudo-MLSE approach described in section III-B to model (51) gives rise, for QR signals, to the three-input pseudo-MLSE receiver. This receiver still generates the sequence $\widehat{\mathbf{b}} \triangleq\left(\widehat{b}_{1}, \ldots, \widehat{b}_{K}\right)$ which minimizes (17) but where $z_{\mathrm{F}}(k)$, now denoted by $z_{\mathrm{F}_{3}}(k)$, is defined by $z_{\mathrm{F}_{3}}(k) \triangleq \Re\left[j^{-k} y_{\mathrm{F}_{3}}(k)\right]$, where $y_{\mathrm{F}_{3}}(k)$ is given by

$$
y_{\mathrm{F}_{3}}(k)=\int \mathbf{g}_{\mathrm{F}_{3}}^{H}(f)\left[\mathbf{R}_{n_{\mathrm{F}_{3}}}^{0}(f)\right]^{-1} \mathbf{x}_{\mathrm{F}_{3}}(f) e^{j 2 \pi f k T} d f .
$$

Here, $\mathbf{R}_{n_{\mathrm{F}_{3}}}^{0}(f)$ is the power spectral density matrix of $\mathbf{n}_{\mathrm{F}_{3}}(t)$, while $r_{k, k^{\prime}}$ is now defined by

$$
r_{k, k^{\prime}}=j^{k^{\prime}-k} \int \mathbf{g}_{\mathrm{F}_{3}}^{H}(f)\left[\mathbf{R}_{n_{\mathrm{F}_{3}}}^{0}(f)\right]^{-1} \mathbf{g}_{\mathrm{F}_{3}}(f) e^{j 2 \pi f\left(k-k^{\prime}\right) T} d f .
$$

Noting that $y_{\mathrm{F}_{3}}(k)$ is the sampled version, at time $t=k T$, of the output of the TI filter whose frequency response is

$$
\mathbf{w}_{\mathrm{F}_{3}}^{H}(f) \triangleq\left(\left[\mathbf{R}_{n_{\mathrm{F}_{3}}}^{0}(f)\right]^{-1} \mathbf{g}_{\mathrm{F}_{3}}(f)\right)^{H},
$$

and whose input is $\mathbf{x}_{\mathrm{F}_{3}}(t)$, the structure of the three-input pseudo-MLSE receiver is then depicted at Figure 5. It is composed of the TI WL filter (54), followed by a sampling at the symbol rate, a derotation operation, a real part capture and a decision box implementing the Viterbi algorithm since $r_{k, k^{\prime}}^{*}=r_{k^{\prime}, k}$.

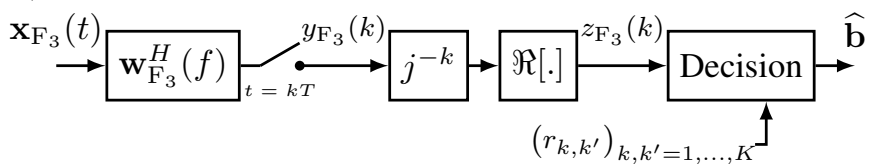

Fig. 5: Structure of the three-input pseudo-MLSE receiver for QR signals

Note that the implementation of the three-input pseudoMLSE receiver requires the knowledge or the estimation of $\mathbf{g}_{\mathrm{F}_{3}}(f)$ and $\mathbf{R}_{n_{\mathrm{F}_{3}}}^{0}(f)$ for each frequency $f$. This implementation is again out of the scope of the paper but it requires the estimation of the channel impulse response of both the SOI and the CCI and the estimation of the background noise power spectral density.

\section{SINR at the output of the three-input pseudo-MLSE re- ceiver}

It is easy to verify from (51), (52) and (53) that $z_{\mathrm{F}_{3}}(n)$ can be written as (21) where $z_{n, \mathrm{~F}}(n)$ is replaced by $z_{n, \mathrm{~F}_{3}}(n)=$ $\Re\left[j^{-n} y_{n, \mathrm{~F}_{3}}(n)\right]$ and where $y_{n, \mathrm{~F}_{3}}(n)$ is defined by (52) for $k=n$ with $\mathbf{n}_{\mathrm{F}_{3}}(f)$ instead of $\mathbf{x}_{\mathrm{F}_{3}}(f)$. The SINR on the current symbol $n$ at the output of the three-input pseudoMLSE receiver is then defined by

$$
\begin{aligned}
\operatorname{SINR}_{\mathrm{F}_{3}}(n) & \triangleq \frac{\pi_{b} r_{n, n}^{2}}{\mathrm{E}\left[z_{n, \mathrm{~F}_{3}}^{2}(n)\right]} \\
& =\frac{2 \pi_{b} r_{n, n}^{2}}{\mathrm{E}\left[\left|y_{n, \mathrm{~F}_{3}}(n)\right|^{2}\right]+(-1)^{n} \Re\left(\mathrm{E}\left[y_{n, \mathrm{~F}_{3}}^{2}(n)\right]\right)} .
\end{aligned}
$$

In the presence of $\mathrm{QR} \mathrm{CCI}$, the total noise, $y_{n, \mathrm{~F}_{3}}(t)$, at the output of (54) is SO cyclostationary, which implies that $\mathrm{E}\left[\left|y_{n, \mathrm{~F}_{3}}(n)\right|^{2}\right]$ and $E\left[y_{n, \mathrm{~F}_{3}}^{2}(n)\right]$ have Fourier series expansions given by (23) and (24) respectively, where $\mathrm{r}_{y_{n_{\mathrm{F}}}}^{\gamma_{i}}(f)$ and $\mathrm{c}_{y_{n_{\mathrm{F}}}}^{\delta_{i}}(f)$ are replaced by $\mathrm{r}_{y_{\mathrm{F}_{\mathrm{F}}}}^{\gamma_{i}}(f)$ and $\mathrm{c}_{y_{\mathrm{F}_{\mathrm{F}}}}^{\delta_{i}}(f)$ respectively. Here, the quantities $\gamma_{i}$ and $\delta_{i}^{3}$ now denote the non-conjugate and conjugate $\mathrm{SO}$ cyclic frequencies of $y_{n, \mathrm{~F}_{3}}(t)$ respectively, whereas $\mathrm{r}_{y_{\mathrm{F}_{3}}}^{\gamma_{i}}(f)$ and $\mathrm{c}_{y_{n_{\mathrm{F}}}}^{\delta_{i}}(f)$ are the Fourier transforms of the first, $\mathrm{r}_{y_{\mathrm{F}_{3}}}^{\gamma_{i}}(\tau)$, and second, $\mathrm{c}_{y_{\mathrm{F}_{\mathrm{F}}}}^{\delta_{i}}(\tau)$, cyclic correlation functions of $y_{n, \mathrm{~F}_{3}}(t)$ for the delay $\tau$, and the cyclic frequencies $\gamma_{i}$ and $\delta_{i}$ respectively. Moreover, as $y_{n, \mathrm{~F}_{3}}(t)$ is the output of the TI filter (54), whose input is $\mathbf{n}_{\mathrm{F}_{3}}(t)$, we can write

$$
\begin{aligned}
& \mathrm{r}_{y_{n}, \mathrm{~F}_{3}}^{\gamma_{i}}(f)=\mathbf{w}_{\mathrm{F}_{3}}^{H}\left(f+\frac{\gamma_{i}}{2}\right) \mathbf{R}_{n_{\mathrm{F}_{3}}}^{\gamma_{i}}(f) \mathbf{w}_{\mathrm{F}_{3}}\left(f-\frac{\gamma_{i}}{2}\right), \\
& \mathrm{c}_{y_{n, \mathrm{~F}_{3}}}^{\delta_{i}}(f)=\mathbf{w}_{\mathrm{F}_{3}}^{H}\left(f+\frac{\delta_{i}}{2}\right) \mathbf{C}_{n_{\mathrm{F}_{3}}}^{\delta_{i}}(f) \mathbf{w}_{\mathrm{F}_{3}}^{*}\left(\frac{\delta_{i}}{2}-f\right),
\end{aligned}
$$

where $\mathbf{R}_{n_{\mathrm{F}_{3}}}^{\gamma_{i}}(f)$ and $\mathbf{C}_{n_{\mathrm{F}_{3}}}^{\delta_{i}}(f)$ are the Fourier transforms of the first, $\mathbf{R}_{n_{\mathrm{F}_{3}}}^{\gamma_{i}}(\tau)$, and second, $\mathbf{C}_{n_{\mathrm{F}_{3}}}^{\delta_{i}}(\tau)$, cyclic correlation matrices of $\mathbf{n}_{\mathrm{F}_{3}}(t)$ for the delay $\tau$ and the cyclic frequency $\gamma_{i}$ and $\delta_{i}$, respectively. Using (53), (23), (24), (56) and (57) into (55), we obtain an alternative expression of (55) given by

$\operatorname{SINR}_{F_{3}}(n)=$

$$
\frac{2 \pi_{b}\left[\int \mathbf{g}_{\mathrm{F}_{3}}^{H}(f) \mathbf{R}_{n_{\mathrm{F}_{3}}}^{0}(f)^{-1} \mathbf{g}_{\mathrm{F}_{3}}(f) d f\right]^{2}}{\left\{\begin{array}{c}
\sum_{\gamma_{i}} e^{j 2 \pi \gamma_{i} n T} \int \mathbf{w}_{\mathrm{F}_{3}}^{H}\left(f+\frac{\gamma_{i}}{2}\right) \mathbf{R}_{n_{\mathrm{F}_{3}}}^{\gamma_{i}}(f) \mathbf{w}_{\mathrm{F}_{3}}\left(f-\frac{\gamma_{i}}{2}\right) d f \\
\left.+(-1)^{n} \Re\left[\sum_{\delta_{i}} e^{j 2 \pi \delta_{i} n T} \int \mathbf{w}_{\mathrm{F}_{3}}^{H}\left(f+\frac{\delta_{i}}{2}\right) \mathbf{C}_{n_{\mathrm{F}_{3}}}^{\delta_{i}}(f) \mathbf{w}_{\mathrm{F}_{3}}^{*} \frac{\delta_{i}}{2}-f\right) d f\right]
\end{array}\right.}
$$

In the presence of CCI having same nature (QR), symbol period and carrier frequency as the SOI, the non-conjugate $\gamma_{i}$ and conjugate $\delta_{i}$ SO cyclic frequencies at the output $y_{n, \mathrm{~F}_{3}}(t)$ of the filter $\mathbf{w}_{\mathrm{F}_{3}}(f)$ are those of the input $\mathbf{n}_{\mathrm{F}_{3}}(t)$ which are from (13) and (14) $\gamma_{i}=\alpha_{i}=i / T$ and $\delta_{i}=\beta_{i}=(2 i+1) / 2 T$, $i \in \mathbb{Z}$. This implies that $\operatorname{SINR}_{\mathrm{F}_{3}}(n)$, given by (58) does not depend on $n$ and is simply denoted by $\operatorname{SINR}_{\mathrm{F}_{3}}$, given by

$$
\operatorname{SINR}_{\mathrm{F}_{3}}=\frac{2 \pi_{b}\left[\int \mathbf{g}_{\mathrm{F}_{3}}^{H}(f) \mathbf{R}_{n_{\mathrm{F}_{3}}}^{0}(f)^{-1} \mathbf{g}_{\mathrm{F}_{3}}(f) d f\right]^{2}}{\left\{\begin{array}{l}
\sum_{\alpha_{i}} \int \mathbf{w}_{\mathrm{F}_{3}}^{H}\left(f+\frac{\alpha_{i}}{2}\right) \mathbf{R}_{n_{i}}^{\alpha_{i}}(f) \mathbf{w}_{\mathrm{F}_{3}}\left(f-\frac{\alpha_{i}}{2}\right) d f \\
+\Re\left[\sum_{\beta_{i}} \int \mathbf{w}_{\mathrm{F}_{3}}^{H}\left(f+\frac{\beta_{i}}{2}\right) \mathbf{C}_{n_{\mathrm{F}_{3}}}^{\beta_{i}}(f) \mathbf{w}_{\mathrm{F}_{3}}^{*}\left(\frac{\beta_{i}}{2}-f\right) d f\right]
\end{array} .\right.}
$$

\section{E. SINR at the output of the three-input pseudo-MLSE receiver} for one CCI

1) Observation model and statistics: Using again the model (30) with $c_{k}=j^{k} d_{k}$, where the total noise $\mathbf{n}(t)$ is composed of a background noise and one multi-user QR CCI having the same symbol period and carrier frequency as the SOI, we have proved with the same approach as in Appendix A, that 
the matrices $\mathbf{R}_{n_{\mathrm{F}_{3}}}^{\alpha_{i}}(f)$ and $\mathbf{C}_{n_{\mathrm{F}_{3}}}^{\beta_{i}}(f)$ appearing in (59) can be written as

$$
\begin{gathered}
\mathbf{R}_{n_{\mathrm{F}_{3}}}^{\alpha_{i}}(f)=\frac{\pi_{d}}{T} \mathbf{g}_{I_{\mathrm{F}_{3}}}\left(f+\frac{\alpha_{i}}{2}\right) \mathbf{g}_{I_{\mathrm{F}_{3}}}^{H}\left(f-\frac{\alpha_{i}}{2}\right) \\
+N_{0} \delta\left(\alpha_{i}\right) \mathbf{I}_{3 N}+N_{0} \delta\left(\alpha_{i}-\frac{1}{T}\right) \mathbf{J}_{1}+N_{0} \delta\left(\alpha_{i}+\frac{1}{T}\right) \mathbf{J}_{1}^{T} \\
\mathbf{C}_{n_{\mathrm{F}_{3}}}^{\beta_{i}}(f)=\frac{\pi_{d}}{T} \mathbf{g}_{I_{\mathrm{F}_{3}}}\left(f+\frac{\beta_{i}}{2}\right) \mathbf{g}_{I_{\mathrm{F}_{3}}}^{T}\left(\frac{\beta_{i}}{2}-f\right) \\
+N_{0} \delta\left(\beta_{i}-\frac{1}{2 T}\right) \mathbf{J}_{2}+N_{0} \delta\left(\beta_{i}+\frac{1}{2 T}\right) \mathbf{J}_{3} .
\end{gathered}
$$

Here, $\mathbf{g}_{I_{\mathrm{F}_{3}}}(f) \triangleq\left[\mathbf{g}_{I}^{T}(f), \mathbf{g}_{I}^{H}(1 / 2 T-f), \mathbf{g}_{I}^{H}(-1 / 2 T-f)\right]^{T}$ whereas $\mathbf{J}_{1}, \mathbf{J}_{2}$ and $\mathbf{J}_{3}$ are the $(3 N \times 3 N)$ matrices defined by

$\mathbf{J}_{1} \triangleq\left[\begin{array}{lll}\mathbf{0}_{N} & \mathbf{0}_{N} & \mathbf{0}_{N} \\ \mathbf{0}_{N} & \mathbf{0}_{N} & \mathbf{I}_{N} \\ \mathbf{0}_{N} & \mathbf{0}_{N} & \mathbf{0}_{N}\end{array}\right] ; \mathbf{J}_{2} \triangleq\left[\begin{array}{lll}\mathbf{0}_{N} & \mathbf{I}_{N} & \mathbf{0}_{N} \\ \mathbf{I}_{N} & \mathbf{0}_{N} & \mathbf{0}_{N} \\ \mathbf{0}_{N} & \mathbf{0}_{N} & \mathbf{0}_{N}\end{array}\right] ; \mathbf{J}_{3} \triangleq\left[\begin{array}{ccc}\mathbf{0}_{N} & \mathbf{0}_{N} & \mathbf{I}_{N} \\ \mathbf{0}_{N} & \mathbf{0}_{N} & \mathbf{0}_{N} \\ \mathbf{I}_{N} & \mathbf{0}_{N} & \mathbf{0}_{N}\end{array}\right]$.

2) Deterministic channels and zero roll-off: Assuming a SRRC pulse shaping filter $v(t)$ with a zero roll-off, deterministic propagation channels with no delay spread such that (37) holds, and denoting by $\mathrm{SINR}_{\mathrm{QR}_{3}}$ the SINR at the output of the 3-input pseudo-MLSE receiver for QR signals, $\mathrm{SINR}_{\mathrm{QR}_{3}}$ can be computed from (37), (38), (54), (58), (59), (60), (61).

When $\left|\alpha_{s I}\right| \neq 1$, assuming a strong CCI $\left(\varepsilon_{I} \gg 1\right)$, we obtain the following expression whose main steps of the proof are given in Appendix C

$\operatorname{SINR}_{\mathrm{QR}_{3}} \approx 2 \varepsilon_{s}\left[1-\left|\alpha_{s I}\right|^{2}\left(\frac{\left(1-\left|\alpha_{s I}\right|^{2}\right)(1+\Gamma)^{2}+(2-\Gamma) \Gamma}{\left(1-\left|\alpha_{s I}\right|^{2}\right)(5+2 \Gamma)+2(2-\Gamma)}\right)\right]$

where $\Gamma \triangleq \cos ^{2}\left(\psi_{s I}\right)+\cos ^{2}\left(\zeta_{s I}\right)$, where we recall that $\psi_{s I} \triangleq$ $\phi_{s I}-\pi \tau_{I} / 2 T$ whereas $\zeta_{s I} \triangleq \phi_{s I}+\pi \tau_{I} / 2 T$.

When $\left|\alpha_{s I}\right|=1$ and $\varepsilon_{I} \gg 1$, (63) reduces to

$$
\begin{array}{r}
\operatorname{SINR}_{\mathrm{QR}_{3}} \approx 2 \varepsilon_{s}\left\{1-\frac{\left[\cos ^{2}\left(\psi_{s I}\right)+\cos ^{2}\left(\zeta_{s I}\right)\right]}{2}\right\} \\
\left(\psi_{s I}, \zeta_{s I}\right) \neq(k \pi, k \pi), \\
\operatorname{SINR}_{\mathrm{QR}_{3}} \approx \frac{\varepsilon_{s}}{\varepsilon_{j}} \quad\left(\psi_{s I}, \zeta_{s I}\right)=(k \pi, k \pi) .
\end{array}
$$

Note that the value of $\operatorname{SINR}_{\mathrm{QR}_{3}}$ for $\left|\alpha_{s I}\right| \neq 1$ (63) reduces to its value for $\left|\alpha_{s I}\right|=1$ (64) except for $\psi_{s I}=\zeta_{s I}=k \pi$. We deduce from (63) that the three-input pseudo-MLSE receiver for QR signals performs MAIC as soon as $\left|\alpha_{s I}\right| \neq 1$, while (64) and (65) show that for $\left|\alpha_{s I}\right|=1$, it performs SAIC as long as $\left(\psi_{s I}, \zeta_{s I}\right) \neq(k \pi, k \pi)$, enlightening its interest. Moreover, comparing (45) and (64), we see that $\mathrm{SINR}_{\mathrm{QR}_{3}} \geq$ SINR $_{\mathrm{QR}_{2}}$ for $\left|\alpha_{s I}\right|=1$, proving the best performance of the three-input pseudo MLSE receiver with respect to the twoinput one. In particular, for $\left|\alpha_{s I}\right|=1$ and synchronous signals $\left(\tau_{I}=0\right),(44)$ and (64) show that $\mathrm{SINR}_{\mathrm{QR}_{3}} \approx \mathrm{SINR}_{\mathrm{R}_{2}}$, proving that the three-input receiver for $\mathrm{QR}$ signals behaves similarly as the two-input receiver for $\mathrm{R}$ signals.

To compare, for $\omega=0$ and $\varepsilon_{I} \gg 1, \mathrm{SINR}_{\mathrm{QR}_{3}}$ with $\mathrm{SINR}_{\mathrm{QR}_{2}}$ and $\mathrm{SINR}_{\mathrm{R}_{2}}$ whatever the value of $\tau_{I}$, we must again adopt a statistical perspective. Consequently, we now assume that $\left|\alpha_{s I}\right|=1, \varepsilon_{I} \rightarrow \infty$ and $\phi_{s I}$ and $\pi \tau_{I} / 2 T$ are independent random variables uniformly distributed on $[0,2 \pi]$. Under these assumptions, we easily deduce from (64) the expectation of $\mathrm{SINR}_{\mathrm{QR}_{3}}$ given by

$$
\mathrm{E}\left[\mathrm{SINR}_{\mathrm{QR}_{3}}\right] \approx \varepsilon_{s},
$$

and we deduce from (50) and (66) that $\mathrm{E}\left[\mathrm{SINR}_{\mathrm{QR}_{2}}\right]<$ $\mathrm{E}\left[\mathrm{SINR}_{\mathrm{QR}_{3}}\right] \approx \mathrm{E}\left[\mathrm{SINR}_{\mathrm{R}_{2}}\right]$ for $\left|\alpha_{s I}\right|=1$, which definitely proves, at least for a zero roll-off, that the three-input pseudoMLSE receiver for QR signals gives similar performance, in the mean, than the two-input pseudo-MLSE receiver for $\mathrm{R}$ signals, hence the great interest of the three-input receiver.

3) Deterministic channels and arbitrary roll-off: To extend the previous results for arbitrary values of both the roll-off $\omega$ and $\varepsilon_{I}$, we still assume that $\phi_{s I}$ and $\pi \tau_{I} / 2 T$ are independent random variables uniformly distributed on $[0,2 \pi]$. Under these assumptions, choosing $\varepsilon_{s}=10 \mathrm{~dB}$, Figure 6 shows, for $\mathrm{R}$ and $\mathrm{QR}$ signals, for $N=1, M=1,2$ for $\mathrm{R}$ signals and $M=1,2,3$ for $\mathrm{QR}$ signals, and for $\omega=0,0.5,1$ the variations of $\mathcal{P}_{\mathrm{F}}(x)$ as a function of $x(\mathrm{~dB})$ for $\varepsilon_{I}=20 \mathrm{~dB}$. To complete these results, Figure 7 shows the same variations in the same context but for $\omega=0$ and several values of $\varepsilon_{I}$ corresponding to $\varepsilon_{I}=10 \mathrm{~dB}, 20 \mathrm{~dB}$ and $30 \mathrm{~dB}$, respectively.

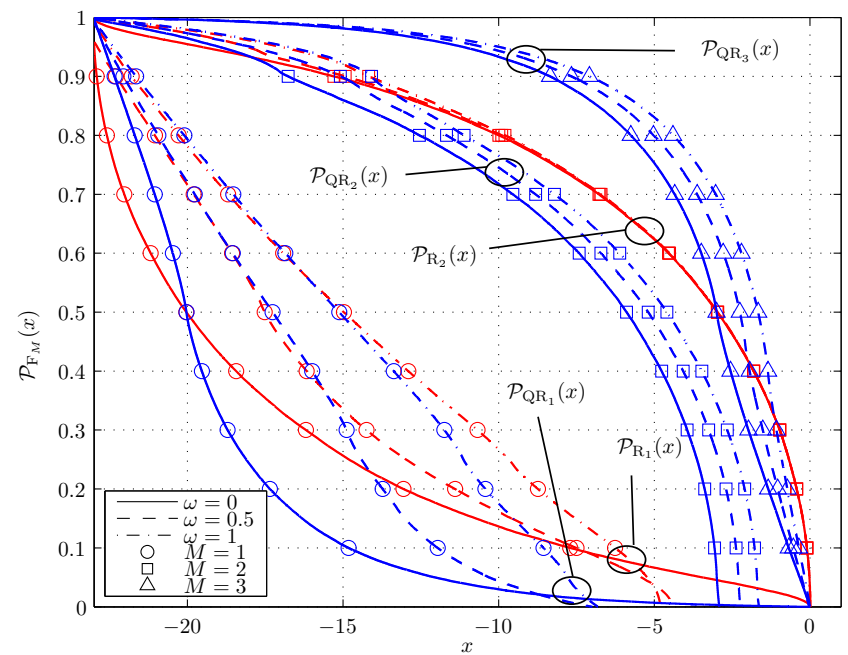

Fig. 6: $\mathcal{P}_{\mathrm{F}_{M}}(x)$ as a function of $x\left(N=1, \varepsilon_{s}=10 \mathrm{~dB}\right.$, $\varepsilon_{I}=20 \mathrm{~dB}, \omega=0,0.5,1$, deterministic one-tap channels, $\mathrm{R}$ and $\mathrm{QR}$ signals)

Note, for QR signals, increasing performance with $\omega$ for $M=2,3$ and the best performance of the three-input receiver with respect to the two-input one whatever $\omega$. Note in particular, for $\omega=0.5, \varepsilon_{I}=20 \mathrm{~dB}$ and $x=-3 \mathrm{~dB}$, that $\mathcal{P}_{\mathrm{QR}_{1}}(x)=\mathcal{P}_{\mathrm{R}_{1}}(x)=0 \%, \mathcal{P}_{\mathrm{QR}_{2}}(x)=26 \%, \mathcal{P}_{\mathrm{R}_{2}}(x)=50 \%$ and $\mathcal{P}_{\mathrm{QR}_{3}}(x)=63 \%$, proving, for $\mathrm{QR}$ signals, the much better performance obtained with $M=3$, instead of $M=2$ and the even better performance obtained, for $x=-3 \mathrm{~dB}$, for $M=3$ with $\mathrm{QR}$ signals, than for $M=2$ with $\mathrm{R}$ signals. Note finally the different distributions of $\mathrm{SINR}_{\mathrm{R}_{2}}$ and $\mathrm{SINR}_{\mathrm{QR}_{3}}$ despite the same expected value for $\omega=0$ and the best performance, whatever the value of $\varepsilon_{I}$, for $M=2$ and R signals with respect to $M=3$ and QR signals when $x$ is close to zero.

4) Rayleigh channels and arbitrary roll-off: The analysis done in sub-section V-E3 for arbitrary values of both the 


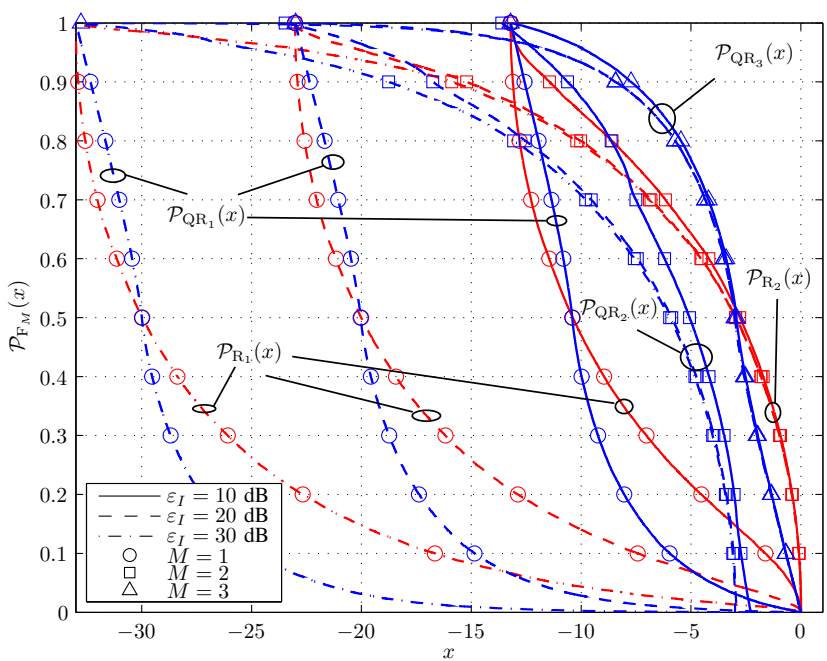

Fig. 7: $\mathcal{P}_{\mathrm{F}_{M}}(x)$ as a function of $x\left(N=1, \varepsilon_{s}=10 \mathrm{~dB}\right.$, $\omega=0, \varepsilon_{I}=10,20,30 \mathrm{~dB}$, deterministic one-tap channels, $\mathrm{R}$ and $\mathrm{QR}$ signals)

roll-off $\omega$ and $\varepsilon_{I}$, is applied in this sub-section, and under the same assumptions, to Rayleigh fading channels instead of deterministic channels and for $\mathrm{R}$ and $\mathrm{QR}$ signals. In this case, each component of $\mathbf{h}$ and $\mathbf{h}_{I}$ are i.i.d. random variables and follows a circular complex Gaussian distribution such that $\varepsilon_{s} \triangleq \pi_{s} \mathrm{E}\left[\mathbf{h}^{H} \mathbf{h}\right] / N_{0}=N \pi_{s} / N_{0}$ and $\varepsilon_{I} \triangleq \pi_{I} \mathrm{E}\left[\mathbf{h}_{I}^{H} \mathbf{h}_{I}\right] / N_{0}=$ $N \pi_{I} / N_{0}$. Under these assumptions, Figure 8 shows the same variations as Figure 6 but for Rayleigh fading channels while Figure 9 reports results analogous to Figure 7 for Rayleigh fading channels and $\varepsilon_{I}=10 \mathrm{~dB}$ and $30 \mathrm{~dB}$. Again these figures show the better performance obtained, for QR signals, with $M=3$ with respect to $M=2$ whatever the value of both the roll-off $\omega$ and $\varepsilon_{I}$ and the even better performance obtained with $M=3$ for $\mathrm{QR}$ signals with respect to $M=2$ for $\mathrm{R}$ ones in most cases.

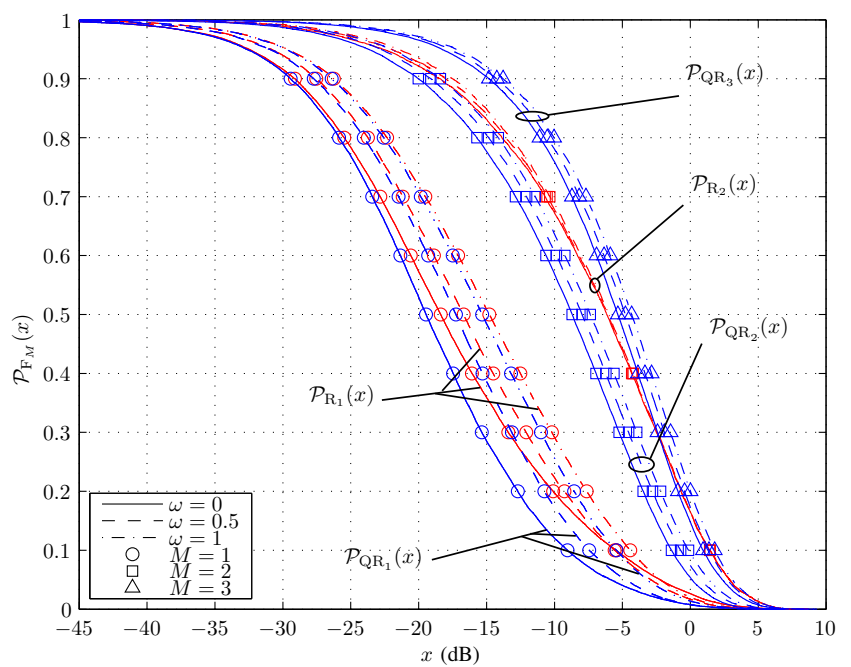

Fig. 8: $\mathcal{P}_{\mathrm{F}_{M}}(x)$ as a function of $x\left(N=1, \varepsilon_{s}=10 \mathrm{~dB}\right.$, $\varepsilon_{I}=20 \mathrm{~dB}, \omega=0,0.5,1$, Rayleigh one-tap channels, R and QR signals)

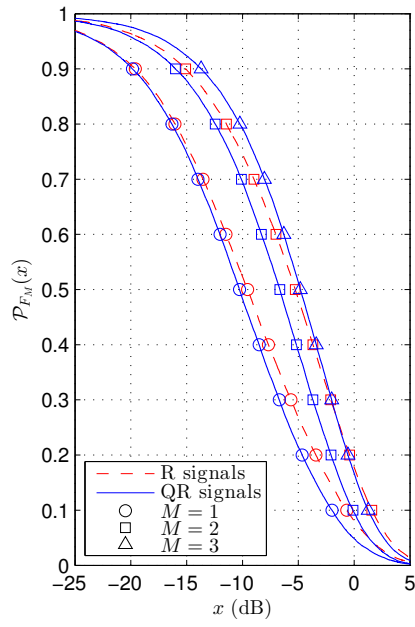

(a) $\varepsilon_{I}=10 \mathrm{~dB}$

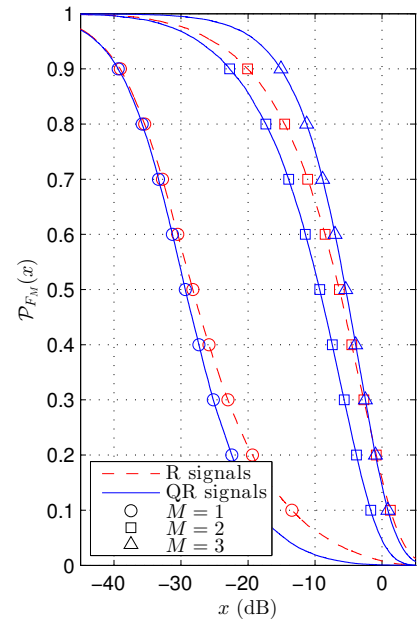

(b) $\varepsilon_{I}=30 \mathrm{~dB}$
Fig. 9: $\mathcal{P}_{\mathrm{F}_{M}}(x)$ as a function of $x\left(N=1, \varepsilon_{s}=10 \mathrm{~dB}\right.$, $\omega=0, \varepsilon_{I}=10,30 \mathrm{~dB}$, Rayleigh one-tap channels, R and $\mathrm{QR}$ signals)

\section{COMPLEXITY ELEMENTS AND OUTPUT SER OF THE PSEUDO-MLSE RECEIVERS FOR ONE CCI}

We give in this section some complexity elements of the $M$-input pseudo-MLSE receiver and we verify that, in the presence of one $\mathrm{CCI}$, the results obtained in section $\mathrm{V}$ through the output SINR criterion are still valid for the output symbol error rate (SER) criterion. To this aim, after giving some insights into the global complexity of the $M$-input pseudoMLSE receiver, we analyse, for both $\mathrm{R}$ and $\mathrm{QR}$ signals, the ISI length the Viterbi algorithm has to take into account at the output of the $M$-input pseudo-MLSE receiver. Finally we present some comparative performance in terms of output SER.

\section{A. Complexity elements of the M-input pseudo-MLSE receiver for one CCI}

The complexity of the $M$-input pseudo-MLSE receiver for one CCI is the sum of three terms. The first one is the complexity required to estimate the global channel impulse responses, $\mathbf{g}(t)$ and $\mathbf{g}_{I}(t)$, of the SOI and CCI respectively, jointly with the estimation of the background noise power spectral density. This first term is not dependent on $M$, is the same for all the receivers for R and QR signals and its computation is out of the scope of the paper. The second term is the complexity required to compute the output of the $M$ input pseudo-matched filter defined by (20) for $M=1,2$ ( $\mathrm{R}$ and $\mathrm{QR}$ signals) and by (54) for $M=3$ (QR signals). This complexity, which depends on $M$, is briefly discussed in this sub-section. The third term is the complexity of the Viterbi algorithm which a priori depends on the signal nature $(\mathrm{R}$ or $\mathrm{QR}$ ) and on $M$ and which is analyzed in the next sub-section. Nevertheless if we take the same Viterbi algorithm for all the receivers, the differential complexity of the receivers is only due to the second term, hence its brief analysis hereafter.

We deduce from (31) and (60) that, for given values of $f$ and $M(M=1,2,3)$, the computation of the power 
spectral density matrix of the extended total noise requires $(M N)^{2}+M N$ complex operations (cops), whereas its inversion requires $8(M N)^{3} / 3$ cops. The product of this matrix inverse with a vector of the same size requires $M N(2 M N-1)$ cops. Thus, for a given value of $f$, the $M$-input pseudomatched filter (20) or (54) requires $(M N)^{2}(3+8 M N / 3)$ cops. In practice (20) and (54) are computed for a given number, $N_{f f t}$, of frequency bins and the associated temporal coefficients are obtained from an inverse FFT. The computation of the $M$-input pseudo-matched filter then requires $N_{f f t}\left[(M N)^{2}(3+8 M N / 3)\right]+M N \times \mathcal{O}(M N \log (M N))$ cops. Finally if we only keep $N_{s}$ temporal samples of this filter, each output of the filter requires $N_{s}\left(2 N_{s}-1\right)$ additional cops. This result shows that the complexity of the $M$-input pseudoMLSE receiver has an order $\mathcal{O}\left(8(M N)^{3} / 3\right)$, without taking into account the Viterbi part.

\section{B. Complexity elements of the Viterbi algorithm for the $M$ - input pseudo-MLSE receivers for one CCI}

It is well-known [54] that the complexity of the Viterbi algorithm is directly linked to both the number of symbols of the constellation and the number of non-zero coefficients $r_{k, k^{\prime}}=r_{k-k^{\prime}}((19)$ and (53)) appearing in the pseudoMLSE metric (17), which both determine the number of states of the algorithm. To compute analytically these coefficients at the output of the $M$-input ( $M=1,2,3)$ pseudo-MLSE receivers considered in this paper, we consider the total noise model (30), we assume a SRRC pulse shaping filter $v(t)$ with a zero roll-off, deterministic propagation channels with no delay spread such that (37) holds and we denote by $r_{k}^{\mathrm{A}_{M}}$ the coefficient $r_{k}$ at the output of the $M$-input pseudo-MLSE receiver for $A(R$ or $Q R)$ signals. Under these assumptions, we obtain the following expressions proved in Appendix D:

$$
\begin{aligned}
r_{k}^{\mathrm{R}_{1}} & =r_{k}^{\mathrm{QR}}=\frac{\mu^{2}\|\mathbf{h}\|^{2}}{N_{0}}\left(1-\left|\alpha_{s I}\right|^{2} \frac{\varepsilon_{I}}{1+\varepsilon_{I}}\right) \delta(k), \\
r_{k}^{\mathrm{R}_{2}} & =\frac{\mu^{2}\|\mathbf{h}\|^{2}}{N_{0}}\left(1-\left|\alpha_{s I}\right|^{2} \frac{\varepsilon_{I}}{1+2 \varepsilon_{I}}\left[1+\cos \left(2 \phi_{s I}\right)\right]\right) \delta(k),(68) \\
r_{k}^{\mathrm{QR}} & =\frac{\mu^{2}\|\mathbf{h}\|^{2}}{N_{0}}\left[2 \delta(k)-\left|\alpha_{s I}\right|^{2} \operatorname{sinc}\left(\frac{k \pi}{2}\right)\left(\frac{(-1)^{k} \varepsilon_{I}}{1+\varepsilon_{I}}\right.\right. \\
& \left.\left.+\frac{\varepsilon_{I}\left(1+\cos \left(2 \psi_{s I}\right)\right)}{1+2 \varepsilon_{I}}\right)\right] \\
r_{k}^{\mathrm{QR}} & =\frac{\mu^{2}\|\mathbf{h}\|^{2}}{N_{0}}\left[3 \delta(k)-\frac{\left|\alpha_{s I}\right|^{2}}{2} \operatorname{sinc}\left(\frac{k \pi}{2}\right)\left(\frac{\varepsilon_{I}\left(1+(-1)^{k}\right)}{1+\varepsilon_{I}}\right.\right. \\
+ & \left.\left.\frac{2 \varepsilon_{I}\left\{\left(1+\cos \left(2 \psi_{s I}\right)\right)+(-1)^{k}\left(1+\cos \left(2 \zeta_{s I}\right)\right)\right\}}{1+2 \varepsilon_{I}}\right)\right] .
\end{aligned}
$$

where $\operatorname{sinc}(x) \triangleq \sin (x) / x$. Expression (67) indicates that $r_{k}^{\mathrm{R}_{1}}=r_{k}^{\mathrm{QR}_{1}}=0$ for $k \neq 0$, which means that for both $\mathrm{R}$ and $\mathrm{QR}$ signals, no ISI is present at the output of the associated conventional receiver. In this case, no Viterbi algorithm is required and the decision is done symbol by symbol. This situation also occurs at the output of the two-input pseudoMLSE receiver for R signals as shown by (68), hence its very simple implementation. However expressions (69) and (70) show that $r_{2 k}^{\mathrm{QR}_{2}}=r_{2 k}^{\mathrm{QR}_{3}}=0$ for $k \neq 0$ but, for $\alpha_{s I} \neq 0$, we obtain in the general case $r_{2 k+1}^{\mathrm{QR}_{2}} \neq 0$ and $r_{2 k+1}^{\mathrm{QR}_{3}} \neq 0$, which shows that ISI is generally present at the output of the $M$-input pseudo-MLSE receiver $(M=2,3)$ for $\mathrm{QR}$ signals and which means that a Viterbi algorithm is required for demodulation. This proves the higher complexity of the two and three-input pseudo-MLSE receiver for QR signals with respect to the twoinput receiver for $\mathrm{R}$ signals and this gives an additional proof of the non-equivalence of $\mathrm{R}$ and $\mathrm{QR}$ signals for the two-input pseudo-MLSE receivers. Moreover, assuming $\left|\alpha_{s I}\right|=1$, a strong interference $\left(\varepsilon_{I} \gg 1\right)$ and $\left(\psi_{s I}, \zeta_{s I}\right) \neq(k \pi, k \pi)$, we obtain from (69) and (70)

$$
\left|\frac{r_{2 k+1}^{\mathrm{QR}_{3}} / r_{0}^{\mathrm{QR}}}{r_{2 k+1}^{\mathrm{QR}} / r_{0}^{\mathrm{QR}}}\right| \approx\left|\frac{\sin ^{2} \psi_{s I}-\sin ^{2} \zeta_{s I}}{\sin ^{2} \psi_{s I}+\sin ^{2} \zeta_{s I}}\right| \leq 1,
$$

which shows, for QR signals, a lower power of ISI in general for the three-input pseudo-MLSE receiver with respect to the two-input pseudo-MLSE receiver, hence the great interest of the former also from a complexity point of view. In particular, in the case of synchronous sources $\left(\tau_{I}=0\right)$, we obtain $\psi_{s I}=$ $\zeta_{s I}$ and $r_{2 k+1}^{\mathrm{QR}_{3}}=0$ whatever the value of $\phi_{s I}$, whereas $r_{2 k+1}^{\mathrm{QR}_{2}} \neq$ 0 for $\phi_{s I} \neq k \pi$. In this latter case, for QR signals, a Viterbi algorithm is required for $M=2$, but not for $M=3$.

Note that expressions (69) and (70), obtained for a zero roll-off, correspond to a worst case for the coefficients $r_{k}^{\mathrm{QR}_{2}}$ and $r_{k}^{\mathrm{QR}_{3}}$ for $k \neq 0$. In practice the roll-off of the SRRC pulse shaping filter is greater than zero and the latter values are lower.

For the computer simulations of the following sub-section, we have constrained the memory of the Viterbi algorithm to be equal to 16 symbols, i.e. we have assumed that $r_{k}^{\mathrm{QR}_{2}}=$ $r_{k}^{\mathrm{QR}_{3}}=0$ for $|k|>8$.

C. Symbol Error Rate at the output of the $M$-input pseudo$M L S E$ receivers for one CCI

1) One Tap deterministic channels: To compare the $M$ input $(M=1,2)$ pseudo-MLSE receivers for $\mathrm{R}$ signals and the $M$-input $(M=1,2,3)$ pseudo-MLSE receivers for $\mathrm{QR}$ signals, from a SER criterion, we consider the transmission of 1000 frames of 184 symbols and we assume, in this subsection, one tap deterministic channels which are constant over a frame and random from a frame to another. For each frame, we assume that $\phi_{s I}$ and $\pi \tau_{I} / 2 T$ are independent random variables uniformly distributed on $[0,2 \pi]$. Under these assumptions, Figure 10 shows the variations of the SER at the output of the considered receivers for both $\mathrm{R}$ and $\mathrm{QR}$ signals, as a function of $\varepsilon_{s}$, for $N=1, \omega=0.5$ and $\varepsilon_{I} / \varepsilon_{s}=10$ $\mathrm{dB}$. Note the poor performance of the conventional receivers $(M=1)$ and the much better performance of the $M$-input receivers for $M>1$. Note also the best performance obtained for $M=3$ for $\mathrm{QR}$ signals, which even outperform the results obtained with $M=2$ for $\mathrm{R}$ signals. This is due to more $\mathrm{SO}$ informations exploited by the 3-input pseudo-MLSE receiver for QR signals with respect to the 2-input pseudo-MLSE receiver for $\mathrm{R}$ signals, jointly with the different distributions of $\mathrm{SINR}_{\mathrm{QR}_{3}}$ and $\mathrm{SINR}_{\mathrm{R}_{2}}$.

2) One Tap Rayleigh channels: To complete the previous results and under the assumptions of Figure 10, Figure 11 


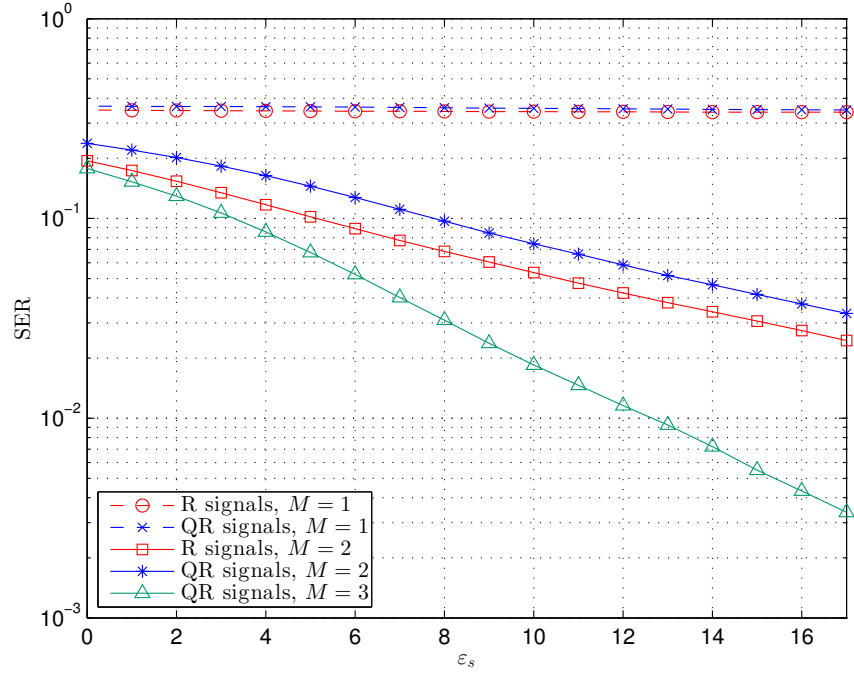

Fig. 10: SER as a function of $\varepsilon_{s}\left(N=1, \varepsilon_{I} / \varepsilon_{s}=10 \mathrm{~dB}\right.$, $\omega=0.5$, deterministic one tap channels, $\mathrm{R}$ and $\mathrm{QR}$ signals)

shows the same variations as Figure 10, but as a function of $\mathrm{E}\left[\varepsilon_{s}\right]=10 \mathrm{~dB}$ for Rayleigh fading channels for which $h$ and $h_{I}$ are circular Gaussian channels, such that $\mathrm{E}\left[\varepsilon_{I}\right] / \mathrm{E}\left[\varepsilon_{s}\right]=10$ dB. The conclusions of Figure 10 hold for Figure 11.

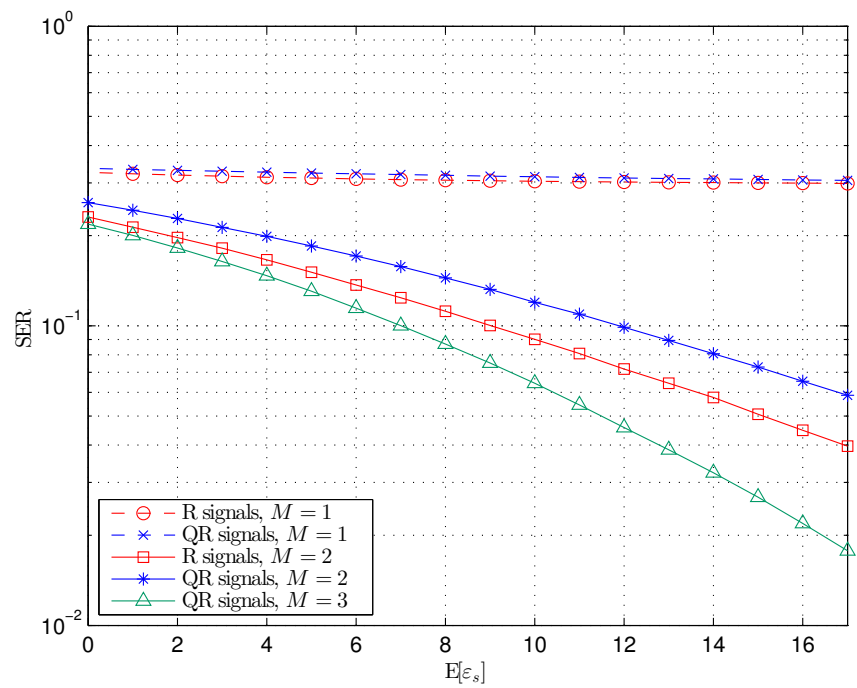

Fig. 11: SER as a function of $\mathrm{E}\left[\varepsilon_{s}\right]\left(N=1, \mathrm{E}\left[\varepsilon_{I}\right] / \mathrm{E}\left[\varepsilon_{s}\right]=10\right.$ $\mathrm{dB}, \omega=0.5$, Rayleigh fading one tap channels, $\mathrm{R}$ and $\mathrm{QR}$ signals)

3) Two-Tap Deterministic channels: Finally, we consider in this sub-section a one-tap deterministic channel for the SOI and a two-tap frequency selective deterministic channel for the CCI such that

$$
\begin{aligned}
\mathbf{h}(t) & =\mu \delta(t) \mathbf{h} \\
\mathbf{h}_{I}(t) & =\mu_{I_{1}} \delta\left(t-\tau_{I_{1}}\right) \mathbf{h}_{I_{1}}+\mu_{I_{2}} \delta\left(t-\tau_{I_{1}}-T\right) \mathbf{h}_{I_{2}},
\end{aligned}
$$

where $\mu_{I_{1}}$ and $\mu_{I_{2}}$ control the amplitudes of the first and second paths of the CCI, whereas $\mathbf{h}_{I_{1}}$ and $\mathbf{h}_{I_{2}}$ correspond to the channel vectors of the latter, such that $\mathbf{h}_{I_{1}}^{H} \mathbf{h}_{I_{1}}=\mathbf{h}_{I_{2}}^{H} \mathbf{h}_{I_{2}}=$ $N$. Under these assumptions and for SRRC pulse shaping filters, it is straightforward to verify that $\pi_{I}=\left(\mu_{I_{1}}^{2}+\mu_{I_{2}}^{2}\right) \pi_{d}$. We consider again the transmission of 1000 frames of 184 symbols, constant channels per frame, random channels from a frame to another, and we assume, for each frame, that $\phi_{s}, \phi_{I_{1}}$, $\phi_{I_{2}}$ and $\pi \tau_{I} / 2 T$ are independent random variables uniformly distributed on $[0,2 \pi]$, where $\phi_{I_{1}}$ and $\phi_{I_{2}}$ are the phases of $\mathbf{h}_{I_{1}}(1)$ and $\mathbf{h}_{I_{2}}(1)$ respectively. Under these assumptions, Figure 12 shows the variations of the SER at the output of the considered receivers for both $\mathrm{R}$ and $\mathrm{QR}$ signals, as a function of $\varepsilon_{s}$, for $N=1, \omega=0.5, \varepsilon_{I} / \varepsilon_{s}=10 \mathrm{~dB}$ and $\mu_{I_{1}}=\mu_{I_{2}}$. The conclusions of Figure 10 hold for Figure 12.

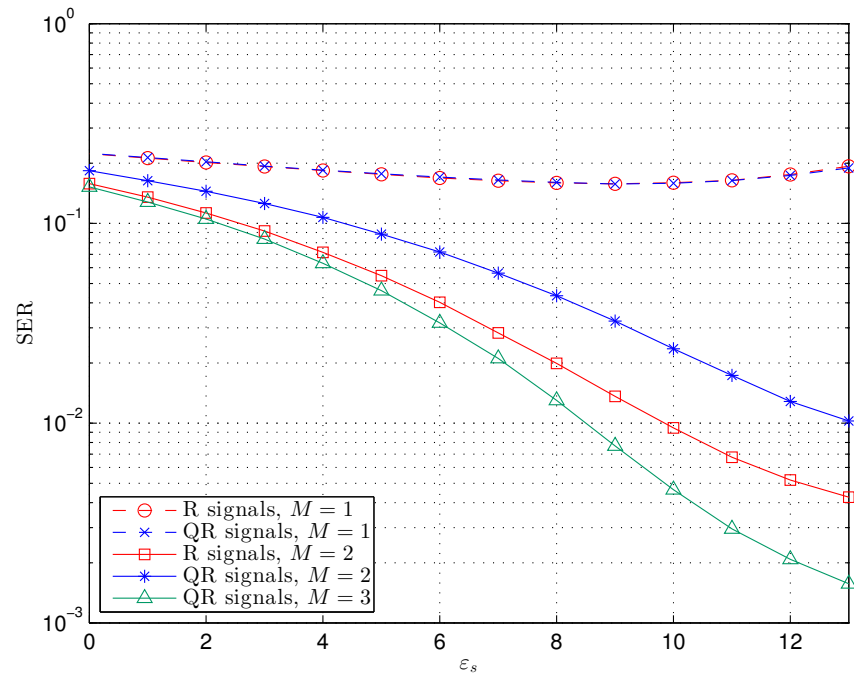

Fig. 12: SER as a function of $\varepsilon_{s}\left(N=1, \varepsilon_{I} / \varepsilon_{s}=10 \mathrm{~dB}\right.$, $\omega=0.5, \mu_{I_{1}}=\mu_{I_{2}}$, deterministic two-tap channels, $\mathrm{R}$ and QR signals)

\section{CONCLUSION}

We have shown in this paper, both analytically and by computer simulations, that contrary to what is accepted as true in the literature, standard (or two-input) WL filtering in the presence of CCI may be less efficient for $\mathrm{QR}$ signals, omnipresent in radiocommunications networks such as GSM, VAMOS or FBMC-OQAM networks, than R ones. This result, which is directly linked to the different SO non-circularity and cyclostationarity properties of these signals, has been proved in this paper using a CT pseudo-MLSE approach for propagation channels with or without delay spread. Such an approach is much more powerful than an MMSE approach, is not dependent of the sample rate of a DT implementation and allows us to develop original analytical performance computations, hence its choice here. Moreover, for both $\mathrm{R}$ and QR signals, the capability of two-input pseudo-MLSE receivers to perform SAIC has been proved for most of frequency selective propagation channels. To improve the standard WL filtering of QR signals in the presence of CCI and to make $\mathrm{QR}$ signals at least almost equivalent to $\mathrm{R}$ ones for WL filtering in such contexts, an enhanced WL receiver has been proposed and analyzed in this paper for arbitrary propagation channels. This enhanced WL receiver is a WL FRESH receiver corresponding to the three-input WL pseudoMLSE receiver. This new receiver has been shown, both analytically and by computer simulations, to be much more powerful than the standard WL receiver for SAIC/MAIC of 
QR signals. Note that these receivers are able to process up to $2 N-1$ CCI from an array of $N$ antennas, hence their interest for many kinds of networks for which the number of CCI may exceed one. The results of the paper, completely new, should open new perspectives and should contribute to develop new powerful WL receivers for CCI mitigation in radiocommunication networks using $\mathrm{QR}$ signals. The main reason for this is that the results of the paper should remain valid not only for other CT approaches, such as MMSE ones, but also for DT approaches. Indeed, as explained in the paper, the reason for the non-equivalence between $\mathrm{R}$ and $\mathrm{QR}$ signals for standard WL filtering is directly related to their different SO cyclostationarity and non-circularity properties and this difference gets beyond the chosen optimization criterion or the kind of implementation (CT or DT). The comparison of R and QR signals for DT WL filtering using MMSE or MLSE criteria, jointly with implementation issues and the role of the oversampling rate, is currently under investigation and will be considered elsewhere. Nevertheless note that preliminary results about the non equivalence between $\mathrm{R}$ and $\mathrm{QR}$ signals for DT WL filtering has already been pointed out in [8] through an MMSE approach.

\section{APPENDIX}

\section{A. Proof of (31) and (32)}

Consider the case of a QR CCI for $M=2$. The other cases are proved similarly. Applying the definitions (2) and (3) to $\widetilde{\mathbf{n}}_{\mathrm{F}}(t) \triangleq\left[\mathbf{n}^{T}(t) e^{-j 2 \pi t / 4 T}, \mathbf{n}^{H}(t) e^{j 2 \pi t / 4 T}\right]^{T}$, we get:

$$
\begin{aligned}
\mathbf{R}_{\widetilde{n}_{\mathrm{F}}}(t, \tau) & =\left[\begin{array}{cc}
\mathbf{R}_{n}(t, \tau) e^{-j 2 \pi \tau / 4 T} & \mathbf{C}_{n}(t, \tau) e^{-j 2 \pi t / 2 T} \\
\mathbf{C}_{n}^{*}(t, \tau) e^{j 2 \pi t / 2 T} & \mathbf{R}_{n}^{*}(t, \tau) e^{j 2 \pi \tau / 4 T}
\end{array}\right] \\
\mathbf{C}_{\widetilde{n}_{\mathrm{F}}}(t, \tau) & =\left[\begin{array}{cc}
\mathbf{C}_{n}(t, \tau) e^{-j 2 \pi t / 2 T} & \mathbf{R}_{n}(t, \tau) e^{-j 2 \pi \tau / 4 T} \\
\mathbf{R}_{n}^{*}(t, \tau) e^{j 2 \pi \tau / 4 T} & \mathbf{C}_{n}^{*}(t, \tau) e^{j 2 \pi t / 2 T}
\end{array}\right]
\end{aligned}
$$

Replacing (4) and (5) into (73) and (74), we derive:

$$
\begin{aligned}
& \mathbf{R}_{\widetilde{n}_{\mathrm{F}}}(t, \tau)= \\
& {\left[\begin{array}{cc}
\sum_{\alpha_{i}} \mathbf{R}_{n}^{\alpha_{i}}(\tau) e^{-j 2 \pi \frac{\tau}{4 T}} e^{j 2 \pi \alpha_{i} t} & \sum_{\beta_{i}} \mathbf{C}_{n}^{\beta_{i}}(\tau) e^{j 2 \pi\left(\beta_{i}-\frac{1}{2 T}\right) t} \\
\sum_{\beta_{i}} \mathbf{C}_{n}^{\beta_{i}{ }^{*}}(\tau) e^{j 2 \pi\left(-\beta_{i}+\frac{1}{2 T}\right) t} & \sum_{\alpha_{i}} \mathbf{R}_{n}^{\alpha_{i}{ }^{*}}(\tau) e^{j 2 \pi^{\frac{\tau}{4 T}}} e^{-j 2 \pi \alpha_{i} t}
\end{array}\right],} \\
& \mathbf{C}_{\widetilde{n}_{\mathrm{F}}}(t, \tau)= \\
& {\left[\begin{array}{cc}
\sum_{\beta_{i}} \mathbf{C}_{n}^{\beta_{i}}(\tau) e^{j 2 \pi\left(\beta_{i}-\frac{1}{2 T}\right) t} & \sum_{\alpha_{i}} \mathbf{R}_{n}^{\alpha_{i}}(\tau) e^{-j 2 \pi \frac{\tau}{4 T}} e^{j 2 \pi \alpha_{i} t} \\
\sum_{\alpha_{i}} \mathbf{R}_{n}^{\alpha_{i}{ }^{*}}(\tau) e^{j 2 \pi \frac{\tau}{4 T}} e^{-j 2 \pi \alpha_{i} t} & \sum_{\beta_{i}} \mathbf{C}_{n}^{\beta_{i}{ }^{*}}(\tau) e^{j 2 \pi\left(-\beta_{i}+\frac{1}{2 T}\right) t}
\end{array}\right],}
\end{aligned}
$$

with $\alpha_{i}=i / T$ and $\beta_{i}=(2 i+1) / 2 T, i \in \mathbb{Z}$. Noting that $\beta_{i}-1 / 2 T=i / T=\alpha_{i}$, (75) and (76) can be rewritten as:

$$
\begin{aligned}
\mathbf{R}_{\widetilde{n}_{\mathrm{F}}}(t, \tau) & =\sum_{\alpha_{i}}\left[\begin{array}{ll}
\mathbf{R}_{n}^{\alpha_{i}}(\tau) e^{-j 2 \pi \frac{\tau}{4 T}} & \mathbf{C}_{n}^{\alpha_{i}+\frac{1}{2 T}}(\tau) \\
\left(\mathbf{C}_{n}^{-\alpha_{i}+\frac{1}{2 T}}(\tau)\right)^{*} & \left(\mathbf{R}_{n}^{-\alpha_{i}}(\tau)\right)^{*} e^{j 2 \pi \frac{\tau}{4 T}}
\end{array}\right] e^{j 2 \pi \alpha_{i} t} \\
& =\sum_{\alpha_{i}} \mathbf{R}_{\tilde{n}_{\mathrm{F}}}^{\alpha_{i}}(\tau) e^{j 2 \pi \alpha_{i} t} \\
\mathbf{C}_{\widetilde{n}_{\mathrm{F}}}(t, \tau) & =\sum_{\alpha_{i}}\left[\begin{array}{cc}
\mathbf{C}_{n}^{\alpha_{i}+\frac{1}{2 T}}(\tau) & \mathbf{R}_{n}^{\alpha_{i}}(\tau) e^{-j 2 \pi_{4} \frac{\tau}{4 T}} \\
\left(\mathbf{R}_{n}^{-\alpha_{i}}(\tau)\right)^{*} e^{j 2 \pi \frac{\tau}{4 T}} & \left(\mathbf{C}_{n}^{-\alpha_{i}+\frac{1}{2 T}}(\tau)\right)^{*}
\end{array}\right] e^{j 2 \pi \alpha_{i} t} \\
& =\sum_{\alpha_{i}} \mathbf{C}_{\widetilde{n}_{\mathrm{F}}}^{\alpha_{i}}(\tau) e^{j 2 \pi \alpha_{i} t}
\end{aligned}
$$

Consequently the Fourier transforms $\mathbf{R}_{\tilde{n}_{\mathrm{F}}}^{\alpha_{i}}(f)$ and $\mathbf{C}_{\widetilde{n}_{\mathrm{F}}}^{\alpha_{i}}(f)$ of $\mathbf{R}_{\widetilde{n}_{\mathrm{F}}}^{\alpha_{i}}(\tau)$ and $\mathbf{C}_{\widetilde{n}_{\mathrm{F}}}^{\alpha_{i}}(\tau)$, respectively, are given by:

$$
\begin{aligned}
& \mathbf{R}_{\widetilde{n}_{\mathrm{F}}}^{\alpha_{i}}(f)=\left[\begin{array}{cc}
\mathbf{R}_{n}^{\alpha_{i}}\left(f+\frac{1}{4 T}\right) & \mathbf{C}_{n}^{\alpha_{i}+1 / 2 T}(f) \\
\left(\mathbf{C}_{n}^{-\alpha_{i}+1 / 2 T}(-f)\right)^{*} & \left(\mathbf{R}_{n}^{-\alpha_{i}}\left(\frac{1}{4 T}-f\right)\right)^{*}
\end{array}\right] \\
& \mathbf{C}_{\widetilde{n}_{\mathrm{F}}}^{\alpha_{i}}(f)=\left[\begin{array}{cc}
\mathbf{C}_{n}^{\alpha_{i}+1 / 2 T}(f) & \mathbf{R}_{n}^{\alpha_{i}}\left(f+\frac{1}{4 T}\right) \\
\left(\mathbf{R}_{n}^{-\alpha_{i}}\left(\frac{1}{4 T}-f\right)\right)^{*} & \left(\mathbf{C}_{n}^{-\alpha_{i}+1 / 2 T}(-f)\right)^{*}
\end{array}\right] .
\end{aligned}
$$

Under the assumption (30), where the first and second cyclospectrum of $\mathbf{n}(t)$, appearing in (79) and (80), are respectively:

$$
\begin{aligned}
\mathbf{R}_{n}^{\alpha_{i}}(f) & =\frac{\pi_{d}}{T} \mathbf{g}_{I}\left(f+\frac{\alpha_{i}}{2}\right) \mathbf{g}_{I}^{H}\left(f-\frac{\alpha_{i}}{2}\right)+N_{0} \delta\left(\alpha_{i}\right) \mathbf{I}_{N}, \\
\mathbf{C}_{n}^{\beta_{i}}(f) & =\frac{\pi_{d}}{T} \mathbf{g}_{I}\left(f+\frac{\beta_{i}}{2}\right) \mathbf{g}_{I}^{T}\left(\frac{\beta_{i}}{2}-f\right),
\end{aligned}
$$

(79) and (80) reduce to (31) and (32), respectively, where $\widetilde{\mathbf{g}}_{I_{\mathrm{F}}}(f)=\left[\mathbf{g}_{I}^{T}\left(f+\frac{1}{4 T}\right), \mathbf{g}_{I}^{H}\left(\frac{1}{4 T}-f\right)\right]^{T}$.

\section{B. Proof of (35)}

Applying the matrix inversion lemma to $\mathbf{R}_{\widetilde{n}_{\mathrm{F}}}^{0}(f)$ deduced from (31):

$$
\mathbf{R}_{\widetilde{n}_{\mathrm{F}}}^{0}(f)^{-1}=\frac{1}{N_{0}}\left[\mathbf{I}_{2 N}-\frac{\widetilde{\mathbf{g}}_{I_{\mathrm{F}}}(f) \widetilde{\mathbf{g}}_{I_{\mathrm{F}}}^{H}(f)}{\left\|\widetilde{\mathbf{g}}_{I_{\mathrm{F}}}(f)\right\|^{2}+\frac{N_{0} T}{\pi_{d}}}\right],
$$

we straightforwardly get for $f \in B_{\mathrm{F}}^{0}$.

$$
\widetilde{\mathbf{g}}_{\mathrm{F}}^{H}(f) \mathbf{R}_{\widetilde{n}_{\mathrm{F}}}^{0}(f)^{-1} \widetilde{\mathbf{g}}_{\mathrm{F}}(f)=\frac{\left\|\widetilde{\mathbf{g}}_{\mathrm{F}}(f)\right\|^{2}}{N_{0}}\left(1-\frac{\left|\widetilde{\alpha}_{S I_{\mathrm{F}}}(f)\right|^{2}}{1+\frac{1}{\widetilde{\varepsilon}_{I_{\mathrm{F}}}(f)}}\right) .
$$

Then, using (20), (83) and (31), we get after some algebra manipulations for $\alpha_{i} \neq 0$ and $f \in B_{\mathrm{F}}^{\alpha_{i}} \cap B_{\mathrm{F}}^{-\alpha_{i}}$ (where $B_{\mathrm{F}}^{\alpha_{i}}$ denotes the set of frequencies $f$ such that $\widetilde{\mathbf{g}}_{\mathrm{F}}\left(f+\frac{\alpha_{i}}{2}\right)$ is nonzero):

$$
\begin{aligned}
& \widetilde{\mathbf{w}}_{\mathrm{F}}^{H}\left(f+\frac{\alpha_{i}}{2}\right) \mathbf{R}_{\widetilde{n}_{\mathrm{F}}}^{\alpha_{i}}(f) \widetilde{\mathbf{w}}_{\mathrm{F}}\left(f-\frac{\alpha_{i}}{2}\right)= \\
& \frac{1}{N_{0}} \frac{\widetilde{\alpha}_{S I_{\mathrm{F}}}\left(f+\frac{\alpha_{i}}{2}\right)\left\|\widetilde{\mathbf{g}}_{\mathrm{F}}\left(f+\frac{\alpha_{i}}{2}\right)\right\|}{\sqrt{\widetilde{\varepsilon}_{I_{\mathrm{F}}}\left(f+\frac{\alpha_{i}}{2}\right)\left(1+\frac{1}{\widetilde{\varepsilon}_{I_{\mathrm{F}}}\left(f+\frac{\alpha_{i}}{2}\right)}\right)}} \\
& \times \frac{\widetilde{\alpha}_{S I_{\mathrm{F}}}^{*}\left(f-\frac{\alpha_{i}}{2}\right)\left\|\widetilde{\mathbf{g}}_{\mathrm{F}}\left(f-\frac{\alpha_{i}}{2}\right)\right\|}{\sqrt{\widetilde{\varepsilon}_{I_{\mathrm{F}}}\left(f-\frac{\alpha_{i}}{2}\right)}\left(1+\frac{1}{\widetilde{\varepsilon}_{I_{\mathrm{F}}}\left(f-\frac{\alpha_{i}}{2}\right)}\right)},
\end{aligned}
$$

whereas from (20)

$$
\widetilde{\mathbf{w}}_{\mathrm{F}}^{H}(f) \mathbf{R}_{\widetilde{n}_{\mathrm{F}}}^{0}(f) \widetilde{\mathbf{w}}_{\mathrm{F}}(f)=\widetilde{\mathbf{g}}_{\mathrm{F}}^{H}(f) \mathbf{R}_{\widetilde{n}_{\mathrm{F}}}^{0}(f)^{-1} \widetilde{\mathbf{g}}_{\mathrm{F}}(f) .
$$

For strong CCI and for which $\widetilde{\varepsilon}_{I_{\mathrm{F}}}(f) \gg 1, \widetilde{\varepsilon}_{I_{\mathrm{F}}}\left(f+\frac{\alpha_{i}}{2}\right) \gg 1$ and $\widetilde{\varepsilon}_{I_{\mathrm{F}}}\left(f-\frac{\alpha_{i}}{2}\right) \gg 1$ for $f \in B_{\mathrm{F}}^{0} \cap B_{\mathrm{F}}^{\alpha_{i}} \cap B_{\mathrm{F}}^{-\alpha_{i}}$ and for frequencies for which $\widetilde{\mathbf{g}}_{I_{\mathrm{F}}}(f)$ is not proportional to $\widetilde{\mathbf{g}}_{\mathrm{F}}(f)$, i.e., such that $\left|\widetilde{\alpha}_{S I_{\mathrm{F}}}(f)\right| \neq 1$, the following approximation is deduced from the comparison between (84) and (85):

$$
\left|\widetilde{\mathbf{w}}_{\mathrm{F}}^{H}\left(f+\frac{\alpha_{i}}{2}\right) \mathbf{R}_{\widetilde{n}_{\mathrm{F}}}^{\alpha_{i}}(f) \widetilde{\mathbf{w}}_{\mathrm{F}}\left(f-\frac{\alpha_{i}}{2}\right)\right| \ll \widetilde{\mathbf{w}}_{\mathrm{F}}^{H}(f) \mathbf{R}_{\tilde{n}_{\mathrm{F}}}^{0}(f) \widetilde{\mathbf{w}}_{\mathrm{F}}(f) .
$$

Furthermore for $f \in B_{\mathrm{F}}^{0}$ and $f \notin B_{\mathrm{F}}^{\alpha_{i}} \cap B_{\mathrm{F}}^{-\alpha_{i}}, \widetilde{\mathbf{w}}_{\mathrm{F}}^{H}(f+$ $\left.\frac{\alpha_{i}}{2}\right) \mathbf{R}_{\tilde{n}_{\mathrm{F}}}^{\alpha_{i}}(f) \widetilde{\mathbf{w}}_{\mathrm{F}}\left(f-\frac{\alpha_{i}}{2}\right)=0$. Consequently as the number of cyclic frequencies $\alpha_{i}$ is finite, due to the limited bandwidth of $v(f),(29)$ reduces to

$$
\operatorname{SINR}_{\mathrm{F}} \approx \pi_{b} \int \widetilde{\mathbf{g}}_{\mathrm{F}}^{H}(f) \mathbf{R}_{\tilde{n}_{\mathrm{F}}}^{0}(f)^{-1} \widetilde{\mathbf{g}}_{\mathrm{F}}(f) d f .
$$


It is easy to verify that for flat fading CCI propagation channels and non-zero CCI, $\widetilde{\mathbf{g}}_{I_{\mathrm{F}}}(f) \neq 0$ for $f \in B_{\mathrm{F}}^{0}$ whereas for frequency selective CCI propagation channels, $\widetilde{\mathrm{g}}_{I_{\mathrm{F}}}(f)$ may be 0 inside $B_{\mathrm{F}}^{0}$ only for discrete values of $f$. Consequently, using (84) into (88), assuming a strong CCI for which $\widetilde{\varepsilon}_{I_{\mathrm{F}}}(f) \gg 1$ when $\widetilde{\varepsilon}_{I_{\mathrm{F}}}(f) \neq 0$ for $f \in B_{f}^{0}$, we obtain the approximation (35) for both $\mathrm{R}$ and $\mathrm{QR}$ strong CCI.

\section{Proof of (63)}

For $v(f)=\sqrt{T} \mathbb{1}_{[-1 / 2 T,+1 / 2 T]}(f)$, the cyclic frequencies reduce to $\alpha_{i} \in\{0,-1 / T,+1 / T\}$ and $\beta_{i} \in\{-1 / 2 T,+1 / 2 T\}$ and (59) can be written as:

$$
\begin{gathered}
\operatorname{SINR}_{\mathrm{F}_{3}}= \\
\int\left[\pi_{b}\left[\int A_{0}(f) d f\right]^{2}\right. \\
\int\left[A_{0}(f)+A_{\frac{-1}{T}}(f)+A_{\frac{1}{T}}(f)\right] d f+\Re\left\{\int\left[B_{\frac{-1}{2 T}}(f)+B_{\frac{1}{2 T}}(f)\right] d f\right\}
\end{gathered}
$$

with

$$
\begin{aligned}
& A_{\alpha_{i}}(f) \triangleq \mathbf{w}_{\mathrm{F}_{3}}^{H}\left(f+\frac{\alpha_{i}}{2}\right) \mathbf{R}_{n_{\mathrm{F}_{3}}}^{\alpha_{i}}(f) \mathbf{w}_{\mathrm{F}_{3}}\left(f-\frac{\alpha_{i}}{2}\right) \\
& B_{\beta_{i}}(f) \triangleq \mathbf{w}_{\mathrm{F}_{3}}^{H}\left(f+\frac{\beta_{i}}{2}\right) \mathbf{C}_{n_{\mathrm{F}_{3}}}^{\beta_{i}}(f) \mathbf{w}_{\mathrm{F}_{3}}^{*}\left(\frac{\beta_{i}}{2}-f\right) .
\end{aligned}
$$

Applying the matrix inversion lemma to $\mathbf{R}_{n_{\mathrm{F}_{3}}}^{0}(f)$ deduced from (60), we straightforwardly get:

$$
A_{0}(f)=\frac{1}{N_{0}}\left(\left\|\mathbf{g}_{\mathrm{F}_{3}}(f)\right\|^{2}-\frac{\left|\mathbf{g}_{\mathrm{F}_{3}}^{H}(f) \mathbf{g}_{I_{\mathrm{F}_{3}}}(f)\right|^{2}}{\left\|\mathbf{g}_{I_{\mathrm{F}_{3}}}(f)\right\|^{2}+\frac{T N_{0}}{\pi_{d}}}\right),
$$

with $\frac{\left\|\mathbf{g}_{\mathrm{F}^{3}}(f)\right\|^{2}}{\mu^{2}\|\mathbf{h}\|^{2}}=\frac{\left\|\mathbf{g}_{\mathrm{I}_{3}}(f)\right\|^{2}}{\mu_{I}^{2}\left\|\mathbf{h}_{I}\right\|^{2}}=v^{2}(f)+v^{2}\left(f-\frac{1}{2 T}\right)+$ $v^{2}\left(f+\frac{1}{2 T}\right) \quad$ and $\quad\left|\mathbf{g}_{\mathrm{F}_{3}}^{H}(f) \mathbf{g}_{I_{\mathrm{F}_{3}}}(f)\right|^{2}=\mu^{2} \mu_{I}^{2}\left|\mathbf{h}^{H} \mathbf{h}_{I}\right|^{2}$ $\left|v^{2}(f)+\left[v^{2}\left(f-\frac{1}{2 T}\right) e^{j 2 \pi \frac{\tau_{I}}{2 T}}+v^{2}\left(f+\frac{1}{2 T}\right) e^{-j 2 \pi \frac{\tau_{I}}{2 T}}\right] e^{-j 2 \phi_{s I}}\right|^{2}$. By integrating $A_{0}(f)$ on $\left[-\frac{1}{T},-\frac{1}{2 T}\right] \cup\left[-\frac{1}{2 T}, 0\right] \cup\left[0, \frac{1}{2 T}\right] \cup$ $\left[\frac{1}{2 T}, \frac{1}{T}\right]$, we obtain after tedious computations:

$$
\int A_{0}(f) d f=\varepsilon_{s}\left[3-\left|\alpha_{s I}\right|^{2}\left(\frac{1}{1+\frac{1}{\varepsilon_{I}}}+\frac{2 \Gamma}{2+\frac{1}{\varepsilon_{I}}}\right)\right],
$$

which gives the approximation

$$
\int A_{0}(f) d f \approx \varepsilon_{s}\left[3-\left|\alpha_{s I}\right|^{2}(1+\Gamma)\right] .
$$

for strong CCI.

Using (60) into (90), the terms $A_{\alpha_{i}}(f)$ for $\alpha_{i}=-\frac{1}{T}$ and $\alpha_{i}=\frac{1}{T}$ can be written as

$$
\begin{aligned}
A_{\alpha_{i}}(f) & =\frac{1}{N_{0}^{2}} \mathbf{g}_{\mathrm{F}_{3}}^{H}\left(f+\frac{\alpha_{i}}{2}\right)\left[\mathbf{I}_{N}-\frac{\mathbf{g}_{I_{\mathrm{F}_{3}}}\left(f+\frac{\alpha_{i}}{2}\right) \mathbf{g}_{I_{\mathrm{F}_{3}}}^{H}\left(f+\frac{\alpha_{i}}{2}\right)}{\left\|\mathbf{g}_{I_{\mathrm{F}_{3}}}\left(f+\frac{\alpha_{i}}{2}\right)\right\|^{2}+\frac{T N_{0}}{\pi_{d}}}\right] \\
& \times\left[\frac{\pi_{d}}{T} \mathbf{g}_{\mathrm{F}_{3}}\left(f+\frac{\alpha_{i}}{2}\right) \mathbf{g}_{I_{\mathrm{F}_{3}}}^{H}\left(f-\frac{\alpha_{i}}{2}\right)+N_{0} \mathbf{J}\left(\alpha_{i}\right)\right] \\
& \times\left[\mathbf{I}_{N}-\frac{\mathbf{g}_{I_{\mathrm{F}_{3}}}\left(f-\frac{\alpha_{i}}{2}\right) \mathbf{g}_{I_{\mathrm{F}_{3}}}\left(f-\frac{\alpha_{i}}{2}\right)}{\left\|\mathbf{g}_{I_{\mathrm{F}_{3}}}\left(f-\frac{\alpha_{i}}{2}\right)\right\|^{2}+\frac{T N_{0}}{\pi_{d}}}\right] \mathbf{g}_{\mathrm{F}_{3}}\left(f-\frac{\alpha_{i}}{2}\right) .
\end{aligned}
$$

where $\mathbf{J}\left(\frac{1}{T}\right) \triangleq \mathbf{J}_{1}$ and $\mathbf{J}\left(-\frac{1}{T}\right) \triangleq \mathbf{J}_{1}^{T}$.

Then using $\mathbf{g}_{I_{\mathrm{F}_{3}}}^{H}\left(f+\frac{\alpha_{i}}{2}\right) \mathbf{J}\left(\alpha_{i}\right) \mathbf{g}_{I_{\mathrm{F}_{3}}}\left(f-\frac{\alpha_{i}}{2}\right)=\left\|\mathbf{g}_{I}(-f)\right\|^{2}$, $\mathbf{g}_{\mathrm{F}_{3}}^{H}\left(f+\frac{\alpha_{i}}{2}\right) \mathbf{J}\left(\alpha_{i}\right) \mathbf{g}_{\mathrm{F}_{3}}\left(f-\frac{\alpha_{i}}{2}\right)=\|\mathbf{g}(-f)\|^{2}$ and $\mathbf{g}_{I_{\mathrm{F}_{3}}}^{H}(f+$ $\left.\frac{\alpha_{i}}{2}\right) \mathbf{J}\left(\alpha_{i}\right) \mathbf{g}_{\mathrm{F}_{3}}\left(f-\frac{\alpha_{i}}{2}\right)=\left[\mathbf{g}_{\mathrm{F}_{3}}^{H}\left(f+\frac{\alpha_{i}}{2}\right) \mathbf{J}\left(\alpha_{i}\right) \mathbf{g}_{\mathrm{F}_{3}}\left(f-\frac{\alpha_{i}}{2}\right)\right]^{*}=$
$\mathbf{g}^{H}(-f) \mathbf{g}_{I}(-f)$ in (95), we obtain for strong CCI with similar tedious computations as for the derivation of (94):

$$
\int\left[A_{\frac{-1}{T}}(f)+A_{\frac{1}{T}}(f)\right] d f \approx 2 \varepsilon_{s}\left(1-\left|\alpha_{s I}\right|\right) .
$$

Then using (61) into (91), for $\beta_{i}=-\frac{1}{2 T}$ and $\beta_{i}=\frac{1}{2 T}$ we prove similarly to the approximation (96):

$$
\int\left[B_{\frac{-1}{2 T}}(f)+B_{\frac{1}{2 T}}(f)\right] d f \approx \varepsilon_{s}\left[4-\left|\alpha_{s I}\right|^{2}(2+\Gamma)\right] .
$$

Plugging the approximations (94), (96) and (97) into (89) completes the proof.

\section{Proof of (67) to (70)}

The coefficients $r_{k}$ are deduced from the expressions of $r_{k^{\prime}, k^{\prime}+k}$ given by (19) for $M=1,2$, for both $\mathrm{R}$ and $\mathrm{QR}$ signals, and by (53) for $M=3$ for QR signals.

Noting that for $v(f)=\sqrt{T} \mathbb{1}_{[-1 / 2 T,+1 / 2 T]}(f)$ the different terms $\left\|\widetilde{\mathbf{g}}_{\mathrm{F}}(f)\right\|^{2},\left\|\widetilde{\mathbf{g}}_{I_{\mathrm{F}}}(f)\right\|^{2}$ and $\left|\widetilde{\mathbf{g}}_{\mathrm{F}}^{H}(f) \widetilde{\mathbf{g}}_{I_{\mathrm{F}}}(f)\right|^{2}$ [resp., $\left\|\mathbf{g}_{\mathrm{F}_{3}}(f)\right\|^{2},\left\|\mathbf{g}_{I_{\mathrm{F}_{3}}}(f)\right\|^{2}$ and $\left.\left|\mathbf{g}_{\mathrm{F}_{3}}^{H}(f) \mathbf{g}_{I_{\mathrm{F}_{3}}}(f)\right|^{2}\right]$ for $M=1,2$ [resp., $M=3$ ] are constant piecewise functions on intervals $[c / 2 T,(c+1) / 2 T], c \in\{-2-1,0,1\}$ and zero elsewhere, for which

$$
\int_{c / 2 T}^{(c+1) / 2 T} T e^{j 2 \pi f k T} d f=\frac{1}{2} e^{j \pi k\left(c+\frac{1}{2}\right)} \operatorname{sinc}\left(\frac{\pi k}{2}\right),
$$

the different coefficients $r_{k}$ (67) to (70) are deduced by straightforward computations.

\section{REFERENCES}

[1] W. M. Brown and R. B. Crane, "Conjugate linear filtering," IEEE Transactions on Information Theory, vol. 15, no. 4, pp. 462-465, Jul. 1969.

[2] W. Gardner, "Cyclic Wiener filtering: theory and method," IEEE Transactions on Communications, vol. 41, no. 1, pp. 151-163, Jan. 1993.

[3] B. Picinbono and P. Chevalier, "Widely linear estimation with complex data," IEEE Transactions on Signal Processing, vol. 43, no. 8, pp. 20302033, Aug. 1995.

[4] P. Chevalier, "Optimal array processing for non-stationary signals," in Proceedings of the IEEE International Conference on Acoustics, Speech, and Signal Processing (ICASSP), vol. 5, May 1996, pp. 2868-2871.

[5] B. Picinbono, "On circularity," IEEE Transactions on Signal Processing, vol. 42, no. 12, pp. 3473-3482, Dec. 1994.

[6] Z. Ding and G. Li, "Single-channel blind equalization for GSM cellular systems," IEEE Journal on Selected Areas in Communications, vol. 16, no. 8, pp. 1493-1505, Oct. 1998.

[7] H. Trigui and D. T. M. Slock, "Performance bounds for cochannel interference cancellation within the current GSM standard," Signal Processing, vol. 80, no. 7, pp. 1335-1346, Jul. 2000.

[8] P. Chevalier and F. Pipon, "New insights into optimal widely linear array receivers for the demodulation of BPSK, MSK, and GMSK signals corrupted by noncircular interferences-application to SAIC," IEEE Transactions on Signal Processing, vol. 54, no. 3, pp. 870-883, Mar. 2006.

[9] R. Meyer, W. Gerstacker, R. Schober, and J. Huber, "A single antenna interference cancellation algorithm for increased GSM capacity," IEEE Transactions on Wireless Communications, vol. 5, no. 7, pp. 1616-1621, Jul. 2006.

[10] A. Mostafa, R. Kobylinski, I. Kostanic, and M. Austin, "Single antenna interference cancellation (SAIC) for GSM networks," in Proceedings of the 58th IEEE Vehicular Technology Conference (VTC-Fall), vol. 2, Oct. 2003, pp. 1089-1093.

[11] L. Popova, R. Meyer, W. H. Gerstacker, W. H. Gerstacker, and W. Koch, "Downlink Performance Improvements in (A)synchronous GPRS Networks using Single/Multiple Antenna Interference Cancellation Techniques," in Proceedings of the 12th European Wireless Conference (EWC), Apr. 2006, pp. 1-7. 
[12] J. Olivier and W. Kleynhans, "Single antenna interference cancellation for synchronised GSM networks using a widely linear receiver," IET Communications, vol. 1, no. 1, pp. 131-136, Feb. 2007.

[13] S. Chen, S. Tan, and L. Hanzo, "Adaptive beamforming for binary phase shift keying communication systems," Signal Processing, vol. 87, no. 1, pp. 68-78, Jan. 2007.

[14] K. Kuchi and V. Prabhu, "Performance evaluation for widely linear demodulation of PAM/QAM signals in the presence of rayleigh fading and co-channel interference," IEEE Transactions on Communications, vol. 57, no. 1, pp. 183-193, Jan. 2009.

[15] _ , "Interference cancellation enhancement through generalized widely linear equalization in QAM systems," IEEE Transactions on Wireless Communications, vol. 8, no. 4, pp. 1585-1590, Apr. 2009

[16] R. Paiva, R. Vieira, R. Iida, F. Tavares, M. Saily, J. Hulkkonen, R. Jarvela, and K. Niemela, "GSM voice evolution using orthogonal subchannels," IEEE Communications Magazine, vol. 50, no. 12, pp. 8086, Dec. 2012.

[17] M. Vutukuri, R. Malladi, K. Kuchi, and R. Koilpillai, "SAIC receiver algorithms for VAMOS downlink transmission," in Proceedings of the 8th International Symposium on Wireless Communication Systems (ISWCS), Nov. 2011, pp. 31-35.

[18] W. Gerstacker, R. Schober, R. Meyer, F. Obernosterer, M. Ruder, and H. Kalveram, "GSM/EDGE: A mobile communications system determined to stay," AEU - International Journal of Electronics and Communications, vol. 65, no. 8, pp. 694-700, Aug. 2011.

[19] M. Ruder, R. Meyer, F. Obernosterer, H. Kalveram, R. Schober, and W. Gerstacker, "Receiver Concepts and Resource Allocation for OSC Downlink Transmission," IEEE Transactions on Wireless Communications, vol. 13, no. 3, pp. 1568-1581, Mar. 2014.

[20] D. Molteni and M. Nicoli, "Joint OSC Receiver for Evolved GSM/EDGE Systems," IEEE Transactions on Wireless Communications, vol. 12, no. 6, pp. 2608-2619, Jun. 2013.

[21] D. Astely, E. Dahlman, A. Furuskar, Y. Jading, M. Lindstrom, and S. Parkvall, "LTE: the evolution of mobile broadband," IEEE Communications Magazine, vol. 47, no. 4, pp. 44-51, Apr. 2009.

[22] D. Bai, C. Park, J. Lee, H. Nguyen, J. Singh, A. Gupta, Z. Pi, T. Kim, C. Lim, M.-G. Kim, and I. Kang, "LTE-advanced modem design: challenges and perspectives," IEEE Communications Magazine, vol. 50, no. 2, pp. 178-186, Feb. 2012

[23] M. Konrad and W. Gerstacker, "Interference Robust Transmission for the Downlink of an OFDM-Based Mobile Communications System," EURASIP Journal on Wireless Communications and Networking, vol. 2008, no. 1, p. 549371, Nov. 2007.

[24] P. Chevalier and F. Dupuy, "Widely Linear Alamouti Receiver for the Reception of Real-Valued Constellations Corrupted by Interferences - The Alamouti-SAIC/MAIC Concept," IEEE Transactions on Signal Processing, vol. 59, no. 7, pp. 3339-3354, Jul. 2011.

[25] G. Wunder et al., "5gnow: non-orthogonal, asynchronous waveforms for future mobile applications," IEEE Communications Magazine, vol. 52, no. 2, pp. 97-105, Feb. 2014

[26] B. Farhang-Boroujeny and R. Kempter, "Multicarrier communication techniques for spectrum sensing and communication in cognitive radios,' IEEE Communications Magazine, vol. 46, no. 4, pp. 80-85, Apr. 2008.

[27] P. Siohan, C. Siclet, and N. Lacaille, "Analysis and design of OFDM/OQAM systems based on filterbank theory," IEEE Transactions on Signal Processing, vol. 50, no. 5, pp. 1170-1183, May 2002.

[28] T. Fusco, L. Izzo, A. Petrella, and M. Tanda, "Blind Symbol Timing Estimation for OFDM/OQAM Systems," IEEE Transactions on Signal Processing, vol. 57, no. 12, pp. 4952-4958, Dec. 2009.

[29] M. Caus and A. I. Perez-Neira, "Comparison of linear and widely linear processing in MIMO-FBMC systems," in Proceedings of the Tenth International Symposium on Wireless Communication Systems (ISWCS), Aug. 2013, pp. 1-5.

[30] M. Caus and A. Perez-Neira, "Multi-Stream Transmission for Highly Frequency Selective Channels in MIMO-FBMC/OQAM Systems," IEEE Transactions on Signal Processing, vol. 62, no. 4, pp. 786-796, Feb. 2014.

[31] Y. Cheng and M. Haardt, "Widely Linear Processing in MIMO FBMC/OQAM Systems," in Proceedings of the Tenth International Symposium on Wireless Communication Systems (ISWCS), Aug. 2013, pp. 1-5.

[32] S. Josilo, M. Narandzic, S. Tomic, and S. Nedic, "Widely linear filtering based kindred co-channel interference suppression in FBMC waveforms," in Proceedings of the 11th International Symposium on Wireless Communications Systems (ISWCS), Aug. 2014, pp. 776-780.

[33] A. Ishaque and G. Ascheid, "Widely linear receivers for SMT systems with TX/RX frequency-selective I/Q imbalance," in Proceedings of the 25th IEEE International Symposium on Personal, Indoor, and Mobile Radio Communication (PIMRC), Sep. 2014, pp. 800-805.

[34] W. Gerstacker, R. Schober, and A. Lampe, "Receivers with widely linear processing for frequency-selective channels," IEEE Transactions on Communications, vol. 51, no. 9, pp. 1512-1523, Sep. 2003.

[35] G. Gelli, L. Paura, and F. Verde, "On the existence of FIR zeroforcing equalizers for nonredundantly precoded transmissions through FIR channels," IEEE Signal Processing Letters, vol. 12, no. 3, pp. 202205, Mar. 2005.

[36] D. Raphaeli, "A Reduced Complexity Equalizer for OQPSK," IEEE Transactions on Communications, vol. 58, no. 1, pp. 46-51, Jan. 2010.

[37] R. Chauvat, P. Chevalier, and J. P. Delmas, "How to Make QuasiRectilinear Signals (MSK, GMSK, OQAM) Almost Equivalent to Rectilinear Ones (BPSK, ASK) for Widely Linear Filtering in the Presence of CCI," in Proceedings of the 19th International ITG Workshop on Smart Antennas WSA, Mar. 2015, pp. 1-6.

[38] P. Chevalier, R. Chauvat, and J. P. Delmas, "Quasi-rectilinear (MSK, GMSK, OQAM) co-channel interference mitigation by three inputs widely linear fresh filtering," in Proceedings of the IEEE International Conference on Acoustics, Speech and Signal Processing (ICASSP), Apr. 2015, pp. 2434-2438.

[39] P. Chevalier and A. Blin, "Widely Linear MVDR Beamformers for the Reception of an Unknown Signal Corrupted by Noncircular Interferences," IEEE Transactions on Signal Processing, vol. 55, no. 11, pp. 5323-5336, Nov. 2007.

[40] J. Yeo and J. H. Cho, "Asymptotic Frequency-Shift Properizer for Block Processing of Improper-Complex Second-Order Cyclostationary Random Processes," IEEE Transactions on Information Theory, vol. 60, no. 7, pp. 4083-4100, Jul. 2014.

[41] H. Wong and J. Chambers, "Two-stage interference immune blind equaliser which exploits cyclostationary statistics," Electronics Letters, vol. 32, no. 19, pp. 1763-1764, Sep. 1996.

[42] G. Gelli, L. Paura, and A. Tulino, "Cyclostationarity-based filtering for narrowband interference suppression in direct-sequence spread-spectrum systems," IEEE Journal on Selected Areas in Communications, vol. 16, no. 9, pp. 1747-1755, Dec. 1998.

[43] G. Latouche, D. Pirez, and P. Vila, "MMSE cyclic equalization," in Proceedings of the IEEE Military Communications Conference (MILCOM), vol. 1, Oct. 1998, pp. 150-154 vol.1.

[44] K. Zhou, S. Cao, R. Song, and L. Zhang, "Some blind FRESH equalization algorithms with anti-interference capabilities," Journal of Electronics (China), vol. 25, no. 6, pp. 768-773, Nov. 2008.

[45] A. Mirbagheri, K. Plataniotis, and S. Pasupathy, "An enhanced widely linear CDMA receiver with OQPSK modulation," IEEE Transactions on Communications, vol. 54, no. 2, pp. 261-272, Feb. 2006.

[46] W. Gardner and C. Reed, "Making the most out of spectral redundancy in GSM: cheap CCI suppression," in Proceedings of the Thirty-Fifth Asilomar Conference on Signals, Systems and Computers, vol. 1, Nov. 2001, pp. 883-889 vol.1.

[47] A. U. H. Sheikh and F. Hendessi, "FRESH-DFE: A New Structure for Interference Cancellation," Wireless Personal Communications, vol. 44, no. 2, pp. 101-118, Jan. 2008.

[48] P. Laurent, "Exact and Approximate Construction of Digital Phase Modulations by Superposition of Amplitude Modulated Pulses (AMP),' IEEE Transactions on Communications, vol. 34, no. 2, pp. 150-160, Feb. 1986.

[49] J. G. Proakis, Digital Communications By John G. Proakis, 4th ed. McGrawHill, 2000.

[50] W. Gardner, W. Brown, and C.-K. Chen, "Spectral Correlation of Modulated Signals: Part II-Digital Modulation," IEEE Transactions on Communications, vol. 35, no. 6, pp. 595-601, Jun. 1987.

[51] P. Gournay and P. Viravau, "Corrélation spectrale théorique des modulations CPM partie I : résultat analytique pour les modulations cpfsk à 2 états (1-rec)," Annales Des Télécommunications, vol. 53, no. 7-8, pp. 267-278, Jul. 1998

[52] D. Vučić and M. Obradović, "Spectral correlation evaluation of MSK and offset QPSK modulation," Signal Processing, vol. 78, no. 3, pp. 363-367, Nov. 1999.

[53] S. Sallem, J.-P. Delmas, and P. Chevalier, "Optimal SIMO MLSE receivers for the detection of linear modulation corrupted by noncircular interference," in Proceedings of the IEEE Statistical Signal Processing Workshop (SSP), Aug. 2012, pp. 840-843.

[54] G. Ungerboeck, "Adaptive Maximum-Likelihood Receiver for CarrierModulated Data-Transmission Systems," IEEE Transactions on Communications, vol. 22, no. 5, pp. 624-636, May 1974. 